\title{
Cardiac Differentiation of Human Pluripotent Stem Cells Using Defined
} Extracellular Matrix Proteins Reveals Essential Role of Fibronectin

5 Jianhua Zhang ${ }^{1,2}$, Ran Tao ${ }^{1}$, Pratik A. Lalit ${ }^{1}$, Juliana L. Carvalho ${ }^{1,3}$, Yogananda Markandeya ${ }^{1}$,

6 Sean P. Palecek ${ }^{4}$, Timothy J. Kamp ${ }^{1,2,5}$

7

8

${ }^{1}$ Department of Medicine, School of Medicine and Public Health, University of Wisconsin-

10 Madison, Madison, WI 53705, USA

$11{ }^{2}$ Stem Cell and Regenerative Medicine Center, University of Wisconsin-Madison, Madison, WI 1253705 , USA

$13{ }^{3}$ Department of Genomic Sciences and Biotechnology, Catholic University of Brasilia, Brasilia, 14 Distrito Federal - BRAZIL

$15{ }^{4}$ Department of Chemical and Biological Engineering, College of Engineering, University of

16 Wisconsin, Madison, WI 53706, USA

${ }^{5}$ Department of Cell and Regenerative Biology, School of Medicine and Public Health, University of Wisconsin-Madison, Madison, WI 53705, USA

Contact Information: Timothy J. Kamp, MD, PhD

8459 WIMR II

1111 Highland Ave.

Madison, WI 53705

Email: tjk@medicine.wisc.edu

Phone: (608) 263-1172

Fax: (608) 263-0405

30 Classification: Biological Sciences/Developmental Biology

31 Key words: human pluripotent stem cells, fibronectin, epithelial-mesenchymal transition,

32 precardiac mesoderm, cardiomyocytes. 


\section{Abstract}

36 Research and therapeutic applications using human pluripotent stem cell-derived cardiomyocytes

37 (hPSC-CMs) require robust differentiation strategies. Efforts to improve hPSC-CM

38 differentiation have largely overlooked the role of extracellular matrix (ECM). The present study

39 investigates the ability of defined ECM proteins to promote hPSC cardiac differentiation.

40 Fibronectin, laminin-111, and laminin-521 enabled hPSCs to attach and expand; however,

41 fibronectin ECM either endogenously produced or exogenously added promoted, while laminins

42 inhibited, cardiac differentiation in response to growth factors Activin A, BMP4, and bFGF.

43 Inducible shRNA knockdown of fibronectin prevented Brachyury ${ }^{+}$mesoderm formation and

44 subsequent hPSC-CM differentiation. Antibodies blocking fibronectin binding to integrin $\beta 1$, but

45 not $\alpha 5$, inhibited cardiac differentiation. Furthermore, inhibition of integrin-linked kinase

46 blocked cardiac differentiation. These results identify fibronectin, laminin-111 and laminin-521

47 as defined substrates enabling cardiac differentiation of hPSCs and uncover the essential role of

48 fibronectin and downstream signaling pathways in the early stage of hPSC-CM differentiation.

\section{$50 \quad$ Introduction}

51 Human pluripotent stem cells (hPSCs) including embryonic stem cells (ESCs) and induced

52 pluripotent stem cells (iPSCs) provide a powerful model system for research and therapeutic

53 applications. Cardiomyocytes (CMs) derived from hPSCs have been widely and increasingly

54 used in research for cardiac development and clinical applications for cardiac repair and

55 regeneration, drug testing and precision medicine. Methods and protocols to differentiate hPSCs

56 to CMs (hPSC-CMs) have been advanced significantly in the past decade. The most investigated

57 cardiac differentiation protocols have focused on using soluble molecules including growth 
58 factors and small molecules to treat hPSCs to promote stage-specific cardiac progenitors and

59 ultimately to differentiate to hPSC-CMs. These protocols also require extracellular matrix

60 (ECM) as the substrate to enable hPSC attachment, survival, proliferation and differentiation.

61 However, the ECM proteins involved in cardiac differentiation of hPSCs and the ECM activated

62 signaling pathways have been far less investigated and elucidated. The ECM composition during

63 development is dynamic and provides key signals contributing to stage-specific transitions [1].

64 Our previous study showed that hPSCs cultured on the commercially available ECM preparation,

65 Matrigel, more efficiently and reproducibly differentiate to hPSC-CMs in response to Activin

$66 \mathrm{~A} / \mathrm{BMP} 4 / \mathrm{bFGF}$ signaling if they concurrently received overlays of Matrigel during the initiation

67 of differentiation, the matrix sandwich protocol [2]. The Matrigel overlays promote the first

68 stage of differentiation, the epithelial to mesenchymal transition to form Brachyury ${ }^{+}$precardiac

69 mesoderm, mimicking primitive streak in development [3]. However, Matrigel is a complex

70 mixture of ECM proteins produced from Engelbreth-Holm-Swarm mouse sarcoma cells, is not

71 fully defined, and exhibits batch-to-batch variability. The essential ECM components responsible

72 for promoting the initial stages of cardiogenesis in the Matrigel sandwich protocol as well as the

73 optimal ECM environment to promote cardiogenesis in general remain to be determined.

75 Complex mixtures of ECM proteins such as Matrigel have enabled attachment and self-renewal

76 of hPSCs in appropriate media, and more recently recombinant ECM proteins and synthetic

77 substrates have been identified that can support long-term culture of hPSCs [4]. These defined

78 substrates mimic the ECM components present in the earliest embryo including laminins,

79 collagens, fibronectin, vitronectin, and proteoglycans. The hPSCs interact with the substrates via

80 the transmembrane receptors, integrins, and cell adhesion molecules, such as cadherins. 
81 However, for cardiac differentiation protocols, a substrate that both allows attachment of the

82 hPSCs and also supports proliferation and subsequent differentiation is needed. Strong signals to

83 maintain self-renewal and pluripotency provided by the ECM will impede the differentiation

84 processes, so a composition of ECM that is dynamic and plays multiple roles in support of hPSC

85 proliferation as well as differentiation is theoretically optimal. Yap and colleagues utilized a

86 combination of recombinant laminins, laminin-521 (LN521) which has been demonstrated to

87 promote self-renewal of hPSCs [5] and laminin-221 (LN221) to enable differentiation to cardiac

88 progenitors [6]. Others using a design of experiment statistical approach found a combination of

89 three ECM proteins optimal for cardiac differentiation of hPSCs, collagen type I, laminin-111

90 (LN111) and fibronectin (FN) [7, 8]. Burridge and colleagues systematically tested a range of

91 different substrates in a defined small molecule-based cardiac differentiation protocol and found

92 a variety of substrates including a synthetic vitronectin-derived peptide, recombinant E-cadherin,

93 recombinant human vitronectin, recombinant human LN521, truncated LN511 and human FN,

94 and a FN mimetic enabled hPSC-CM differentiation [9]. However, these important studies have

95 not examined the impact of dynamic manipulation of defined ECM proteins or ECM signaling

96 pathways in cardiac differentiation, nor characterized the changes in endogenous ECM proteins

97 that ultimately contribute to the cellular transitions. In the present study, we tested a variety of

98 human recombinant and defined ECM proteins for both attachment and overlay of hPSC cultures

99 in the matrix sandwich protocol and investigated the ECM activated signaling pathways. We

100 found that of the tested ECM proteins, only FN overlays promoted cardiac differentiation

101 comparable to Matrigel overlays; in contrast, LN111, LN521 and collagen IV (COL4) overlays

102 inhibited cardiogenesis. Furthermore, hPSCs differentiated efficiently to hPSC-CMs without

103 overlay when grown on FN, LN111, and LN521. Regardless of the ECM preparation used as the 
bioRxiv preprint doi: https://doi.org/10.1101/2021.04.09.439173; this version posted April 11, 2021. The copyright holder for this preprint (which was not certified by peer review) is the author/funder, who has granted bioRxiv a license to display the preprint in perpetuity. It is made available under aCC-BY 4.0 International license.

104 attachment substrate, we identified an essential role of FN promoting the initial stages of

105 differentiation acting via transmembrane integrin $\beta 1$ receptors and potential downstream

106 integrin-linked kinase (ILK) signaling. 


\section{Results}

108 Defined extracellular matrix proteins support hPSC adhesion, growth and cardiac

\section{9 differentiation}

110 We tested whether defined human ECM proteins could replace Matrigel in the matrix sandwich

111 cardiac differentiation protocol. The matrix sandwich protocol uses Matrigel coating for hPSC

112 adhesion and expansion followed by overlaying the proliferating hPSCs with Matrigel at day -2

113 and day 0 of differentiation with the addition of growth factors Activin A at day 0-1, followed by

114 addition of BMP4 and bFGF at day 1-5 (Fig. 1A). Therefore, we first tested the ability of defined

115 ECM proteins to support hPSC adhesion and expansion. DF19-19-11T iPSCs or H1 ESCs were

116 seeded on human LN111, LN521, COL4, and FN coated surfaces and cultured in mTeSR1

117 medium. The hPSCs grew as a monolayer on LN111, LN521, and FN and exhibited similar

118 morphology and expression of pluripotency markers of OCT4 and SSEA4 as hPSCs grown on

119 Matrigel (Fig. 1B and Supplementary Fig. S1). However, the hPSCs seeded on COL4 did not

120 grow as a confluent monolayer (Supplementary Fig. S2), so this matrix coating was not tested

121 further.

123 For those matrix substrates which supported monolayer growth of hPSCs, including LN111,

124 LN521 and FN, we tested overlay of defined ECM proteins in the matrix sandwich protocol.

125 Cardiac differentiation was measured by flow cytometry for $\mathrm{cTnT}^{+}$cells at 15 days of

126 differentiation (Fig. 1C and Supplementary Fig. S3). DF19-9-11T iPSCs seeded on Matrigel

127 coated surface showed poor cardiac differentiation in response to the growth factors without an

128 overlay of matrix, but with Matrigel overlays the percentage of $\mathrm{cTnT}^{+}$cells was significantly

129 increased as we previously reported [2]. Interestingly, FN overlays were as effective as Matrigel 
130 overlays in promoting cardiac differentiation of the hPSCs growing on Matrigel. However, if

131 cells were seeded on LN111, LN521, or FN coated surfaces, the overlay of Matrigel or FN did

132 not further increase the efficiency of hPSC-CM generation, and overlays of LN111, LN521 and

133 COL4 strongly inhibited cardiac differentiation. These results demonstrated that the defined

134 ECM proteins of LN111, FN and to a lesser extent LN521 support hPSC adhesion, growth and

135 cardiac differentiation in monolayer hPSC culture and do not require a matrix overlay for

136 efficient cardiac differentiation using the Activin A/BMP4/bFGF growth factor-directed

137 protocol.

hPSCs monolayer culture on LN111 substrate promoted endogenous FN production

140 Because FN promoted cardiac differentiation as coated matrix protein as well as an overlay

141 matrix protein and because FN plays key roles in EMT during gastrulation and cardiogenesis [3,

142 10-21], we next examined for the presence of FN ECM in hPSC culture. DF19-9-11T iPSCs

143 plated on the Matrigel-coated surface and cultured in mTeSR1 per the matrix sandwich protocol

144 were immunolabeled with a FN antibody on day $-3,-2,-1$ and 0 without permeabilizing the cells

145 to examine extracellular FN protein (Fig. 2A). On day -3 and -2 , minimal immunolabeled $\mathrm{FN}$ in

146 ECM was observed. However, after 4 days of culture (day 0) immunolabeled fibrillar FN ECM

147 was abundant in the matrix sandwich culture (Fig. 2A, B). In contrast, the monolayer culture

148 control in the absence of Matrigel overlay had significantly less FN ECM present by day 0 (Fig.

$1492 \mathrm{~A}, \mathrm{~B})$. This suggests that the Matrigel overlay promotes production of endogenous FN or

150 remodeling of FN ECM relative to the monolayer culture control without Matrigel overlay.

151 Because hPSC cultured on LN111 coated surface without matrix overlay enabled efficient

152 cardiac differentiation (Fig. 1C), we examined the endogenous FN production in the hPSC 
153 culture on LN111 coated surface in which no exogenous FN was added. DF19-9-11T iPSCs

154 growing on LN111 coated surface without any matrix overly were immunolabeled with the FN

155 antibody without permeabilizing the cells. Confocal z-scan of the cell culture showed no

156 detectable FN ECM at day -3 and -2, similar to the Matrigel/Matrigel sandwich culture; however,

157 by day 0, dense fibrillary FN ECM was present in the cell culture on LN111 coated surface (Fig.

158 2C). Similar to our previous study of the Matrigel/Matrigel sandwich culture [2], the hPSCs

159 growing on the LN111 coated surface without any matrix overlay formed multilayer cultures as

160 shown in the side view of the confocal z-scan as did FN/FN matrix sandwich culture (Fig. 2D).

161 To determine if the hPSC culture results in accumulation of endogenously produced laminin

162 ECM as well, DF19-9-11T iPSCs cultured on FN, LN111 and LN521 coated surfaces were

163 immunolabeled with an antibody detecting laminins without permeabilizing the cells, and did not

164 show measurable laminin ECM after 4 days of growth on these defined matrices (Supplementary

165 Fig. S4). These results together with the above cardiac differentiation results supported the

166 potential role of $\mathrm{FN}$ in promoting ActivinA/BMP4/bFGF directed hPSC cardiac differentiation.

168 Differentiation of hPSCs on LN111 substrate undergo EMT and generate precardiac

169 mesoderm in FN rich ECM

170 To characterize the early stages of cardiac differentiation of hPSCs cultured on LN111 and

171 treated with Activin A/BMP4/bFGF signaling, we examined markers of EMT, mesoderm and

172 cardiac mesoderm. Gene expression was assessed by quantitative RT-PCR, and upon the

173 addition of Activin A, BMP4 and bFGF, there was significant upregulation of transcription

174 factors associated with EMT including SNAI1 [22], SNAI2 [23] and TWIST [24] (Fig. 3A). The

175 mesenchymal cell markers of vimentin (VIM), fibronectin $(F N 1)$ and $\mathrm{N}$-cadherin $(C D H 2)$ were 
176 also greatly upregulated by day 3 (Fig. 3A). In contrast, E-cadherin expression (CDH1), an

177 epithelial cadherin, was greatly downregulated by day 3 of differentiation. The

178 mesendoderm/precardiac mesoderm transcription factors GSC, MIXL1, SOX17, TBXT and

179 MESP1 were transiently upregulated followed by expression of cardiac transcription factors of

180 ISL1, NKX2-5 and GATA4 at day 3-5 (Fig. 3B).

182 To determine if FN ECM persisted or remodeled during the early stages of cardiac differentiation

183 on LN111 substrate, immunolabeling of the early differentiated DF19-9-11T iPSCs on day 0-3

184 for FN and Brachyury was performed. Confocal z-scan imaging showed abundant fibronectin

185 ECM at each day, and Brachyury ${ }^{+}$cells were associated with the dense network of FN ECM

186 (Fig. 3C), suggesting that the Brachyury ${ }^{+}$cells interact with FN. Similar Brachyury ${ }^{+}$cells and the

187 FN ECM network were also observed in the Matrigel/Matrigel and FN/FN matrix sandwich

188 cultures (Supplementary Fig. S5). Together, these results show that hPSCs grown on LN111,

189 undergo the early stages of cardiac differentiation with transitions to mesoderm and cardiac

190 mesoderm progenitors occurring in an endogenously generated FN rich ECM, similarly as in the

191 Matrigel/Matrigel and FN/FN matrix sandwich cultures.

193 FN is essential for cardiac differentiation of hPSCs

194 To determine if $\mathrm{FN}$ is essential for precardiac mesoderm formation in our protocol and to

195 investigate the stage-specific roles of FN during cardiac differentiation of hPSCs, we generated a

196 doxycycline inducible FN knockdown system using FN1 shRNA (Supplementary Fig. S6). The

197 two vectors shown in Fig. 4A were incorporated into lentivirus and transduced into hPSCs.

198 Clones were selected by neomycin resistance from both hESC line H1 and hiPSC line DF19-9-

199 11T. To confirm the doxycycline inducibility of the FN1 shRNA in the cell lines, we first 
examined for doxycycline-induced bicistronic mCherry (Supplementary Fig. S7A). Inducible FN knockdown was demonstrated by immunolabeling with FN antibody (Supplementary Fig. S7B) and quantitative western-blot for FN expression (Supplementary Fig. S7C, D).

204 Cardiac differentiation was performed using the monolayer based protocol with hPSCs growing

205 on LN111 coated surface and treated with growth factors of Activin A, BMP4 and bFGF as

206 shown in Fig. 4(B) To probe the stage-specific effect of FN knockdown during cardiac

207 differentiation, dox was added at different time points: day 0-1, day 1-5, and day 5-7, and cardiac

208 differentiation was measured by flow cytometry for $\mathrm{cTnT}^{+}$cells at 15 days of differentiation.

209 Cardiac differentiation was significantly inhibited when FN was knocked down at day 0-1,

210 whereas FN knockdown at day 1-5, or day 5-7, did not have significant impact on the $\mathrm{cTnT}^{+}$

211 cells when compared to the no dox control (Fig. 4C).

213 Because FN knockdown at day 0-1 significantly inhibited cardiac differentiation, we next tested

214 if exogenous $\mathrm{FN}$ at this stage can rescue cardiac differentiation. Using the same protocol as

215 shown in Fig. 4B with dox induction of FN knockdown at day 0-1, we added soluble human FN

$216\left(3 \mathrm{ug} / \mathrm{cm}^{2}\right)$ in the cell culture on day 0-1. Cells were differentiated for 15 days, and cardiac

217 differentiation was measured by flow cytometry for $\mathrm{cTnT}^{+}$cells as above. Adding exogenous $\mathrm{FN}$

218 fully rescued the cardiomyocyte differentiation, giving rise to similar percentage of $\mathrm{cTnT}^{+}$cells

219 compared to the no FN knockdown control (Fig. 4C). However, adding exogenous FN (3ug/cm²)

220 at day 1-5 or day 5-7 did not significantly increase the percentage of $\mathrm{cTnT}^{+}$cells when compared

221 to the no dox control at day 1-5 and day 5-7, respectively (Fig. 4C). Furthermore, the dox

222 induction of FN knockdown is concentration dependent (Supplementary Fig. S8). With the dox

223 concentration higher than $4 \mathrm{ug} / \mathrm{ml}$, it caused significant cell death after 24 hours and no cells 
224 survived by 15 days of differentiation, and the cardiac differentiation could not be rescued with

225 adding exogenous FN, indicating the toxicity of doxcycline to the cells (Supplementary Fig.

226 S8A). We confirmed the detrimental effect of the high concentration of dox when added at day

227 0-1 during the cardiac differentiation with the regular hPSC line in which high concentration of

228 dox caused significant cell death and no cells survived after 2 days of differentiation

229 (Supplementary Fig. S8B).

\section{FN is required for formation of Brachyury ${ }^{+}$cells}

232 Because FN knockdown at day 0-1 dramatically inhibited cardiac differentiation which could be

233 rescued by exogenous FN, we first evaluated the expression of EMT genes which mark the initial

234 transition of hPSCs to mesoderm over the first two days of differentiation. Quantitative RT-PCR

235 was performed at 0, 24, 36 and $48 \mathrm{~h}$ after hPSCs differentiation was initiated (Fig. 5A). The effect

236 of dox-induced knockdown of FN1 transcripts was confirmed by the greater than $50 \%$ reduction

237 in mRNA levels in the FN knockdown and FN knockdown + exogenous FN cell samples at 24h

238 relative to the no dox control (Fig. 5A). By 36h, FN1 transcripts recovered to the control level

239 for FN knockdown condition or were significantly increased in the FN knockdown + exogenous

240 FN samples after dox was removed at 24h. By 48h, there were similar level of FN1 transcripts in

241 both the control and the FN rescue samples, but no cells survived in the FN knockdown group

242 (Fig. 5A). The key EMT transcription factors upregulated during gastrulation [3, 20, 25, 26],

243 SNAI1 and SNAI2, were examined. Quantitative RT-PCR showed SNAI1 expression increased

244 significantly in both the FN knockdown and FN knockdown + exogenous FN samples compared

245 to the control at $24 \mathrm{~h}$, and its expression continuously upregulated in the FN knockdown sample

246 by $36 \mathrm{~h}$. By $48 \mathrm{~h}$ there were similar level of $S N A I 1$ expression in both the control and the FN 
rescue samples; whereas, $S N A I 2$ expression was not significantly different between the groups

248 but gradually upregulated in all three groups in the time course (Fig. 5A). VIM expression,

249 similar to SNAII expression, was increased significantly in the FN knockdown cells by $36 \mathrm{~h}$

250 compared to the control (Fig. 5A), which is consistent with this mesenchymal marker and known

251 target of SNAII.

253 We next examined the mesendodermal/precardaic mesodermal progenitors generated in the

254 initial differentiation stage by flow cytometry. The cell counts of the attached cells on day 0-5 of

255 differentiation showed a great reduction of cell number at day 1 in all three groups (Fig. 5B);

256 however, the cells in the control and the $\mathrm{FN}$ rescue groups rapidly proliferated after day 1 . In

257 contrast, no cells survived in the FN knockdown group after day 2 (Fig. 5B). Because Brachyury

258 and Sox 17 are both expressed in mesendodermal progenitors, we co-labeled the cells with

259 Brachyury and Sox 17 antibodies on day 0-5 and analyzed by flow cytometry. Brachyury ${ }^{+}$cells

260 started to emerge at day 1 in all three groups. By day 2, 96-98\% of the cells were Brachyury ${ }^{+}$in

261 both the control and FN rescue groups, but there were no surviving cells in the FN knockdown

262 samples (Fig. 5C). The fraction of cells that were Brachyury ${ }^{+}$rapidly decreased after day 2 in

263 both the control and FN rescue groups, and by day 5 there were minimal Brachyury ${ }^{+}$or Sox $17^{+}$

264 cells present in both groups (Fig. 5C). We performed the same experiment using the DF19-9-11T

265 FN knockdown clones and observed similar results (Supplementary Fig S9). In contrast to the

266 day 0-1 treatment with dox, a later timed dox pulse from day 1-5 did not alter the abundance of

267 Brachyury $^{+}$cells in all three groups over the same time course of differentiation (Supplementary

268 Fig. S10). Together these results suggest that knockdown of FN at day 0-1 does not stop the

269 initiation of EMT upon addition of Activin A at d0 [3, 20], but it prevents the generation and/or 
270 survival of Brachyury ${ }^{+}$precardiac mesodermal progenitors. In contrast, shRNA knockdown of

271 FN at later time points in the protocol did not impact the fate of the differentiating cells.

272

273 FN acts via integrin $\beta 1$ and integrin-linked kinase signaling to promote cardiac

\section{4 differentiation}

275 To investigate the downstream signaling pathways contributing to FN's essential role in 276 formation of precardiac mesoderm, we investigated integrin binding and integrin-linked kinase

277 (ILK) signaling. FN preferentially interacts with $\alpha 5 \beta 1, \alpha v \beta 1$ and $\alpha v \beta 3$ integrin heterodimers on

278 the surface of cells $[27,28]$. We first tested blocking integrin $\alpha 5$ with a monoclonal antibody,

279 P1D6, or integrin $\beta 1$ with a monoclonal antibody, P5D2, at day -2 or day 0 in the matrix

280 sandwich protocol (Fig. 6A). Adding the antibody P1D6 that binds integrin $\alpha 5$ at day -2 or day 0

281 when Matrigel overlays were applied did not block cardiac differentiation as measured by flow

282 cytometry of the $\mathrm{cTnT}^{+}$cells (Fig. 6B). However, adding antibody P5D2 that binds integrin $\beta 1$ at 283 day 0 significantly blocked cardiac differentiation (Fig. 6B). Furthermore, the P5D2 antibody

284 resulted in concentration-dependent inhibition of cardiomyocyte differentiation with the highest

285 concentration tested $(5 \mathrm{ug} / \mathrm{ml})$ completely blocking cardiomyocyte differentiation (Fig. 6C).

287 Integrin-linked kinase (ILK) is a serine/threonine protein kinase which interacts with the 288 cytoplasmic domain of $\beta 1$ and $\beta 3$ integrins and plays crucial roles in transducing signals

289 from ECM components and growth factors to downstream signaling components [29-31]. The

290 kinase activity of ILK is stimulated rapidly and transiently by the engagement of integrins to FN

291 extracellular matrix, as well as by insulin via receptor tyrosine kinases (RTKs), in a PI3K

292 dependent manner [30]. To determine if ILK signaling is involved in the initiation of cardiac 
293 differentiation, we tested the specific ILK inhibitor, Cpd22, in the matrix sandwich protocol.

294 Cpd22 was added at day 0 at different concentrations of $0,1,3$ and $10 \mu \mathrm{M}$ and cells were treated

295 for 24 hours in the matrix sandwich protocol as shown in Fig. 6(A) Cardiac differentiation was

296 measured by flow cytometry of the $\mathrm{cTnT}^{+}$cells at 15 days of differentiation. Cpd22 showed

297 significant inhibition of cardiac differentiation and in a concentration-dependent manner (Fig.

298 6D), and in multiple cell lines (Supplementary Fig. S10).

Because ILK can directly phosphorylate GSK3 and inhibit its kinase activity which modulates

301 Wnt/beta-catenin signaling [30], we sought to confirm the role of ILK in initiation of the cardiac

302 differentiation in a distinct hPSC-CM differentiation protocol based on biphasic modulation of

303 Wnt signaling by small molecules. This protocol involves the activation of canonical Wnt

304 signaling by inhibition of GSK3 $\beta$ to promote Brachyury ${ }^{+}$precardiac mesoderm formation,

305 followed by inhibition of Wnt to promote hPSC-CM differentiation (GiWi protocol) [32, 33].

$306 \mathrm{Cpd} 22$ was added at day 0 at different concentrations of $0,1,3$ and $10 \mu \mathrm{M}$ and cells were treated

307 for 24 hours in the GiWi protocol as shown in Fig. 6E. Cardiac differentiation was measured by

308 flow cytometry of the $\mathrm{cTnT}^{+}$cells at 15 days of differentiation. Cpd22 showed significant

309 inhibition of cardiac differentiation in a concentration-dependent manner in the GiWi protocol

310 (Fig. 6F), similarly to the Matrix Sandwich protocol (Fig. 6C). Together these results are

311 consistent with FN contributing to precardiac mesoderm formation and cardiac differentiation by

312 acting via integrin $\beta 1$ and ILK signaling. 
315 Here we identify defined ECM proteins including LN111, LN521 and FN that can support the

316 attachment of hPSCs and subsequent differentiation into hPSC-CMs in coordination with Activin

$317 \mathrm{~A} / \mathrm{BMP} 4 / \mathrm{bFGF}$ signaling. In addition, we demonstrate that initiating differentiation process with

318 an overlay of defined ECM protein can either promote (FN) or inhibit (LN111, LN521, and

319 COL4) cardiogenesis. Regardless of seeding substrate, cardiac differentiation starts with the

320 generation of Brachyury ${ }^{+}$cells whose survival is dependent on adequate FN in the ECM either

321 generated endogenously or provided exogenously. FN interacts with integrins on the hPSCs

322 including integrin $\beta 1$ potentially activating ILK signaling required for the formation of

323 Brachyury $^{+}$cells. These results provide new insights into defined matrices for cardiac

324 differentiation of hPSCs and the essential role of FN in the earliest stages of differentiation.

The Activin A/BMP4/bFGF directed hPSC cardiac differentiation protocols were initially

327 developed using the embryoid body (EB) approach starting with suspended aggregates of hPSCs

328 [34-36]. Using the combination of Activin A/BMP4/bFGF at the initial differentiation stage in

329 the protocol induces mesoendoderm formation consistent with known signaling pathways during

330 embryonic development [37-40]. However, this growth factor-directed cardiac differentiation

331 exhibited variability when applied either in the EB type protocols or monolayer-based hPSC

332 differentiation approaches $[2,35,41]$. The lack of spatial organization and morphogen gradients

333 in these in vitro differentiation approaches relative to embryonic development likely contribute

334 to the variability. To address this variability, we developed the matrix sandwich method to

335 promote the monolayer-based, growth factor-directed cardiac differentiation of hPSCs [2].

336 Although the matrix sandwich method promoted the EMT during the initial differentiation stage

337 which is required for Brachyury ${ }^{+}$mesoderm formation, the mechanism was unclear due to the 
complex mixture of ECM proteins in Matrigel. In the present study, we demonstrate that the

339 accumulation and assembly of endogenously produced FN ECM induced by Matrigel overlays is

340 a critical enabling feature of the protocol to promote formation of Brachyury ${ }^{+}$precardiac

341 mesoderm and successful cardiac differentiation.

343 A rapid increase in FN ECM is a well described feature of metazoan gastrulation in studies from

344 sea urchin to mouse [42-44]. Fn null mouse embryos generate mesoderm but have profound

345 heart developmental defects with early embryonic lethality [43]. FN interacts with cells by

346 binding integrin receptors activating ILK signaling cascades including PI3K/Akt, growth

347 factor/RTKs, GSK3 and canonical Wnt/ $\beta$-catenin signaling pathways to regulate cell survival,

348 proliferation, migration, and differentiation [30, 31]. Consistent with these developmental studies

349 in model organisms, our data for hPSCs differentiation demonstrate that FN is critical to the

350 genesis and survival of Brachyury ${ }^{+}$precardiac mesodermal cells. FN is proposed to exert its

351 effect by binding to integrin $\beta 1$ and activating ILK signaling which can impact cell survival via

$352 \mathrm{PI} 3 \mathrm{~K} / \mathrm{Akt}$ signaling as well as cell transitions by inhibition of GSK3 to activate canonical Wnt

353 signaling, which enabled the initial precardaic mesoderm formation and subsequent hPSC-CM

354 differentiation in the Activin A/BMP4/bFGF growth factor-mediated differentiation protocol.

356 Over expression of ILK in epithelial cells downregulates E-cadherin, stimulates fibronectin

357 matrix assembly and induces EMT, as well as induces the translocation of $\beta$-catenin to the

358 nucleus, leading to the subsequent activation of the $\beta$-catenin/Tcf transcription complex, the

359 downstream components of the Wnt signaling pathway [31, 45, 46]. We demonstrate that

360 inhibition of ILK significantly inhibited cardiac differentiation in both the growth factor and 
361 small molecule directed protocols. The mechanism of inhibition of cardiac differentiation in the

362 growth factor-directed, matrix sandwich protocol is likely due to the lack of Wnt/ $\beta$-catenin

363 signaling induced by ILK-GSK3 $\beta$ cascades signaling. On the other hand, the mechanism of

364 inhibiting cardiac differentiation in the GiWi protocol is due to the dominant-negative effect of

365 inhibiting ILK resulting in increased GSK3 activity [30].

367 The overall role of the ECM in development and promoting cell fate specification remains an

368 important area for future investigation given the limited understanding. In addition to providing

369 direct signaling such as FN interaction with integrins, mechanical signals from the ECM as well

370 as the topography of the ECM directly impact the biology. For example, ECM stiffness has been

371 suggested to be a key determinant of lineage commitment [47]. The role of the myriad of

372 nonstructural matricellular proteins remain largely unknown. Furthermore, the ECM can provide

373 a reservoir for more soluble signaling molecules such as TGF- $\beta$ [48]. Remarkable remodeling

374 and changes in composition of the ECM have been identified in development, but detailed

375 understanding will require further investigation. hPSC models provide tool to investigate the role

376 of ECM in development and differentiation, but investigations will need to consider both

377 exogenous ECM substrates provided as well as endogenously generated ECM, e.g. the

378 endogenously produced $\alpha-5$ Laminin by undifferentiated hPSCs has been shown to promote

379 hPSC self-renewal [49].

381 Overall, our study identifies several defined ECM proteins, including LN111, LN521, and FN,

382 that can be used successfully for seeding and differentiating hPSCs to CMs. Regardless of the

383 seeding substrate, $\mathrm{FN}$ in the ECM at a critical level as well as integrin $\beta 1$ binding are required to 
384 stimulate the ILK signaling for successful formation of precardiac mesodermal cells required to

385 start the cardiogenesis process whether using the Activin A/BMP4/bFGF protocol or a small

386 molecule protocol based on biphasic Wnt modulation. Ultimately, the choice of ECM substrate

387 used for cardiac differentiation protocols will be based not only on effective outcomes but also

388 on the cost and availability of these defined substrates. The refinement of cardiac differentiation

389 protocols is of growing importance as applications using hPSC-CMs expand including clinical

390 applications.

Materials and Methods

\section{Human PSC culture}

395 Human ESC line H1 and iPSC line DF19-9-11T were used in this study. Human PSCs were 396 cultured on Matrigel (GFR, BD Biosciences) coated 6-well plates in mTeSR1 medium. Cells 397 were passaged using Versene solution (Gibco) every 4-5 days as previously described ([2].

\section{Cardiac differentiation of hPSCs}

400 Human PSCs were dissociated with $1 \mathrm{ml} /$ well Versene solution at $37^{\circ} \mathrm{C}$ for 5 minutes, and 401 seeded on Matrigel, LN111 (BioLamina, 1ug/cm²), LN521 (BioLamina, 0.5ug/cm²) and FN

402 (Corning 354008, 2.5ug/ $\mathrm{cm}^{2}$ ) coated 12-well plates at the density of $6 \times 10^{5}$ cells/well in mTeSR1

403 medium supplemented with $10 \mu \mathrm{M}$ ROCK inhibitor (Y-27632, Tocris). The medium was

404 changed daily. For cardiac differentiation with the matrix sandwich protocol as shown in Fig.

$4051 \mathrm{~A}$, the tested matrix proteins were overlaid on the hPSC culture after $2(\mathrm{~d}-2)$ and 4 (d0) days of 406 seeding when the monolayer of cells reached $80 \%$ and $100 \%$ confluence. The amount of matrix 
407 proteins in overlay was: $8.7 \mathrm{ug} / \mathrm{cm}^{2}$ Matrigel; LN111, LN521, FN and COL4 was $0.3 \mathrm{ug} / \mathrm{cm}^{2}$. For

408 cardiac differentiation with the monolayer based protocol, the matrix overlay was not added. The

409 cells were fed with fresh mTeSR1 medium from d-3 to d0. At d0 the medium was changed to

410 RPMI+B27 without insulin and supplemented with growth factors. The concentration of growth

411 factors were: Activin A (R\&D, 100ng/ml), BMP4 (R\&D, 2.5ng/ml) and bFGF (Waisman

412 Biomanufacturing, UW-Madison,10ng/ml). The volume of medium was $0.8 \mathrm{ml}$ at $\mathrm{d} 0-\mathrm{d} 1$ and

$4131.5 \mathrm{ml}$ at $\mathrm{d} 1-\mathrm{d} 5$. The medium was changed to RPMI+B27 with insulin, and cells were fed every

414 other day and cultured until d15 for flow cytometry analysis. For cardiac differentiation with the

415 GiWi protocol, human PSCs were dissociated with $1 \mathrm{ml} /$ well Versene solution at $37^{\circ} \mathrm{C}$ for 5

416 minutes, and seeded on Matrigel coated 12-well plates at the density of $6-10 \times 10^{5}$ cells/well in

417 mTeSR1 medium supplemented with $10 \mu \mathrm{M}$ ROCK inhibitor (Y-27632). Cells were cultured in

418 mTeSR1 medium with medium change daily until reached $100 \%$ confluence. At d0, the medium

419 was changed to $1 \mathrm{~mL}$ RPMI+B27 without insulin and supplemented with $12 \mu \mathrm{M}$ CHIR99021

420 (Tocris), and changed to $2 \mathrm{~mL}$ RPMI+B27 without insulin after $24 \mathrm{~h} .72 \mathrm{~h}$ after addition of

421 CHIR99021, a combined medium was prepared by collecting $1 \mathrm{~mL}$ of medium from the wells

422 and mixing with same volume of fresh RPMI+B27 without insulin medium and supplemented

423 with $5 \mu \mathrm{M}$ IWP2 (Tocris). The medium was changed to $2 \mathrm{~mL}$ RPMI+B27 without insulin at d5,

424 and to RPMI+B27 with insulin starting from d7. Cells were fed every other day and cultured

425 until d15 for flow cytometry analysis.

\section{Generation of inducible FN1 knockdown hPSC clones}

428 Human PSCs maintained in mTeSR1 medium were used for lentivirus transfection. The cloning 429 strategy was schemed in Supplementary Fig. S12. 
431 HEK 293 TN cells (SBI) were used for lentivirus production. Briefly, 4.5 x $10^{6}$ cells were plated

432 in a $10 \mathrm{~cm}$ dish on the day before transfections. Lipofectamine 2000 (Invitrogen) was used for

433 transfections (1:2 ratio). Transfection conditions used were $-7 \mathrm{ug}$ lentivirus plasmid, 10ug

434 psPAX2 (Addgene plasmid \#12260 - packaging), 5ug pMD2.G (Addgene plasmid \#12259 -

435 envelope). Transfection media was incubated for 15-16 hours, after which it was replaced with

$4365 \mathrm{mls}$ of mTeSR1medium. Lentivirus supernatant was collected after 48-52 hours, filtered (0.45

$437 \mathrm{uM}-$ Millipore) and frozen down at $-80^{\circ} \mathrm{C}$. Lentivirus supernatants were thawed in $37^{\circ} \mathrm{C}$ water

438 bath immediately before infection.

$439 \quad$ hPSCs transfection and neomycin selection

440 Human PSCs at the $80 \%$ confluency after split were incubated with the lentivirus in mTeSR1

441 medium in the presence of 8ug/ml Polybrene (Sigma) for 24-42 hours. After lentivirus

442 transfection, hPSCs were washed with PBS and recovered in mTeSR1 medium for 2-3 days with

443 medium change daily, cells were split once during this recovery. The concentrations of G418

444 (Life technologies) for neo-resistant selection for hPSC lines were determined by the kill curve

445 (Supplementary Fig. S13). G418 (100mg/ml) were diluted in mTeSR1 medium with the final

446 concentrations of $100 \mathrm{ug} / \mathrm{ml}$ for H1 ES cells and 75ug/ml for DF19-9-11T, and hPSCs were under

447 neomycin selectin up to 11 days before single cell isolation and cloning.

\section{$448 \quad$ Single cell isolation and cloning}

449 Human PSCs resistant to neomycin were incubated with Accutase (Gibco) in $37^{\circ} \mathrm{C}$ for $5-10$

450 minutes, and resuspended in mTeSR1 medium to examine under microscope for single cells. If

451 cells were still clustered, cells were spun down and washed with PBS, and underwent Accutase

452 treatment for another time as above. Single cells were plated in 6-well Matrigel coated plate at 
453 the very low density of 50-100 cells $/ \mathrm{cm}^{2}$ with $10 \mathrm{uM}$ Rock inhibitor (Y-27632). Single cells were

454 growing in mTeSR1 medium for 4-7 days, clones were picked up by P200 mircropipette tips and

455 transferred to microtubes with 30ul Versene solution (pre-warmed at room temperature), and

456 incubated at $37^{\circ} \mathrm{C}$ for 5 minutes. Cells were pipette up and down several times and equally

457 dispersed in two wells of Matrigel coated 24-well plates in mTeSR1 medium.

458 Doxycycline induction

459 To induce FN1 shRNA and mCherry expression, doxycycline (Sigma, 8ug/ml) in mTeSR1

460 medium was added to the hPSC clones culture in the 12-well plate for 2-3 days. mCherry

461 expression were examined with EVOS microscope (Life Technologies); FN1 expression were

462 examined by quantitative RT-PCR.

$463 \quad$ Clones expansion and cryopreservation

464 Selected FN1 knockdown positive clones were expanded in mTeSR1 medium in 6-well culture.

465 Cells were cryopreserved in $90 \% \mathrm{FBS}, 10 \% \mathrm{DMSO}$ and $10 \mathrm{uM}$ ROCK inhibitor in liquid nitrogen

466 freezer (LABS20K, Taylor-Wharton).

\section{$468 \quad$ Flow cytometry}

469 Cells were detached from cell culture plates by incubation with $0.25 \%$ trypsin-EDTA

470 (Invitrogen) plus 2\% chick serum (Sigma) for 5 minutes at $37^{\circ} \mathrm{C}$. Cells were vortexed to disrupt

471 the aggregates followed by neutralization by adding equal volume of EB20 medium.[50] Cells

472 were fixed in $1 \%$ paraformaldehyde at $37^{\circ} \mathrm{C}$ water bath for 10 minutes in the dark, permeabilized

473 in ice-cold 90\% methanol for 30 minutes on ice. Cells were washed once in FACS buffer (PBS

474 without $\mathrm{Ca} / \mathrm{Mg}^{2+}, 0.5 \% \mathrm{BSA}, 0.1 \% \mathrm{NaN}_{3}$ ) plus $0.1 \%$ Triton, centrifuged, and supernatant

475 discarded leaving about $50 \mu \mathrm{l}$. For labeling cTnT, the primary antibody was diluted in $50 \mu 1$ 
476 /sample FACS buffer plus $0.1 \%$ Triton and aliquoted to each sample for a total sample volume of

$477100 \mu$ l. Samples were incubated with the primary antibodies overnight at $4^{\circ} \mathrm{C}$. For co-labeling of

478 Brachyury and Sox 17, the cells were incubated with the conjugated antibodies for half an hour at

479 room temperature in dark. Cells were washed once with $3 \mathrm{ml} \mathrm{FACS} \mathrm{buffer} \mathrm{plus} 0.1 \%$ Triton and

480 resuspended in $300-500 \mu \mathrm{FACS}$ buffer plus Triton for analysis. Please refer to Supplementary

481 Material for detail of the primary antibodies. For the secondary antibody labeling for cTnT, cells

482 were washed once with $3 \mathrm{ml} \mathrm{FACS}$ buffer plus $0.1 \%$ Triton after the primary antibody labeling,

483 centrifuged, and supernatant discarded leaving $\sim 50 \mu$ l. Secondary antibody specific to the

484 primary IgG isotype was diluted in FACS buffer plus Triton in a final sample volume of $100 \mu \mathrm{l}$

485 at 1:1000 dilution. Samples were incubated for 30 minutes in the dark at room temperature,

486 washed in FACS buffer plus Triton and resuspended in $300-500 \mu$ FACS buffer plus Triton for

487 analysis. Data were collected on a FACSCaliber flow cytometer (Beckton Dickinson) and

488 analyzed using FlowJo.

\section{Immunocytochemistry}

491 For imaging with EVOS microscope (Life technologies), hPSCs were cultured either in 6-well

492 plates, or seeded and differentiated in 12-well plates, coated with Matrigel or the ECM proteins.

493 For imaging with confocal microscope (Leica), cells were seeded or plated on glass coverslips

494 coated with Matrigel or the ECM proteins. Cells were fixed with 4\% paraformaldehyde for

49515 minutes at room temperature for labeling with different markers. For intracellular markers,

496 cells were permeabilized in $0.2 \%$ Triton X-100 (Sigma) for 1 hour at room temperature. For

497 labeling the ECM proteins, cells were not permeabilized. After fix and permeablization, samples

498 were blocked with 5\% non-fat dry milk (Bio-Rad) in $0.2 \%$ Triton X-100 solution and incubated 
499 for 2 hours at room temperature on a rotator followed by two washes with PBS. Primary

500 antibodies (please refer to Supplementary Material for detail of the primary antibodies) were

501 added in $1 \%$ BSA in PBS solution with or without $0.1 \%$ Triton X-100 depends on the markers to

502 label, and incubated overnight at $4^{\circ} \mathrm{C}$. Samples were washed with $0.2 \%$ Tween 20 in PBS twice

503 and $1 \mathrm{X}$ PBS twice. Secondary antibody specific to the primary IgG isotype were diluted (1:1000)

504 in the same solution as the primary antibodies and incubated at room temperature for 1.5 hours in 505 dark on a rotator. Samples were washed with $0.2 \%$ Tween 20 in PBS twice and 1X PBS twice.

506 Nuclei were labeled with Hoechst or DAPI. Confocal images were analyzed with the Leica LAS

507 AF Lite software.

509 Quantification of fibronectin immuno-fluorescence by ImageJ analysis

510 The fibronectin immuno-fluorescence images of d-3, d-2, d-1, d0 matrix sandwich culture, and

$511 \mathrm{~d} 0$ monolayer culture were evenly split into 4 squares with online image splitting tool

512 (https://www.imgonline.com.ua/eng/cut-photo-into-pieces.php), and imported to ImageJ Fiji

513 software. The control images without primary antibody labeling were also imported and

514 analyzed in the same way by ImageJ Fiji software. The gray value was used to represent the

515 intensity of fluorescence, ranging from 0 (black, low intensity) to 256 (white, high intensity). For

516 mean and standard deviation of gray values, the entire area of each image was selected, and the

517 values were retrieved by selecting Menu bar $>$ Analyze $>$ Measure. The mean gray value was

518 generated from ImageJ by summing each pixel's gray value divided by the number of pixels in

519 the selected area. The final mean fluorescence value was obtained by subtracting the mean gray

520 value of the control image from fibronectin immuno-labeled images. The mean of fluorescence 
521 intensity of each quartiles from each biological replicate in each group were represented in the

522 box plots using Origin v9.

524 RT-PCR and quantitative RT-PCR

525 Cell samples were collected using $0.25 \%$ trypsin-EDTA (Invitrogen) to remove the cells from

526 cell culture plates. Total RNA was purified using QIAGEN RNeasy® Mini kit. Possible genomic

527 DNA contamination was removed by RNase-Free DNase Set (QIAGEN) with the RNeasy

528 columns, or by DNase I (Invitrogen) treatment for 15 minutes at room temperature. $500 \mathrm{ng}$ of

529 total RNA was used for Oligo(dT)20 - primed reverse transcription using SuperScript ${ }^{\mathrm{TM}}$ III First-

530 Strand Synthesis System (Invitrogen). Quantitative RT-PCR was performed using Taqman PCR

531 Master Mix and Gene Expression Assays (Applied Biosystems, Supplementary Table 1) in

532 triplicate for each sample and each gene. $0.5 \mu$ of cDNA from RT reaction was added as

533 template for each Q-PCR reaction. The expression of genes of interest was normalized to that of

534 GAPDH. RT-PCR was carried out using Platinum ${ }^{\mathrm{TM}}$ Taq DNA Polymerase (Invitrogen) or

535 Gotaq Master Mix (Promega) and then subjected to 2\% agarose gel electrophoresis. PCR

536 conditions included denaturation at $94^{\circ} \mathrm{C}$ for 30 seconds, annealing at $60^{\circ} \mathrm{C}$ for 30 seconds, and

537 extension at $72^{\circ} \mathrm{C}$ for 1 minute, for 35 cycles, with $72^{\circ} \mathrm{C}$ extension for 7 minutes at the end.

$538 A C T B$ ( $\beta$-actin) was used as an endogenous control.

\section{Antibody blocking and small molecule inhibition}

541 Blocking antibodies without sodium azide were used for the blocking experiments. Blocking

542 antibody for integrin $\beta 1$, P5D2 (Developmental Studies Hybridoma Bank), and for integrin $\alpha 5$,

543 P1D6 (Developmental Studies Hybridoma Bank), and ILK inhibitor Cpd22 (EMD Millipore) 
544 were diluted in the media to make the final concentrations and added to the culture at the time

545 points as shown in Fig. 6.

\section{$547 \quad$ Statistics}

548 Data are presented as mean \pm standard error of the mean (SEM). Technical and biological

549 replicates are as indicated for each dataset. For datasets with normal distributions, statistical

550 significance was determined by Student's t-test for two groups or one-way ANOVA for multiple

551 groups with post-hoc test using Bonferroni and Tukey methods. Statistical analysis was

552 performed using Origin, v9, $\mathrm{P}<0.05$ was considered statistically significant.

\section{ACKNOWLEDGMENTS}

555 The authors thank Dr. Deane Mosher for the consultation of fibronectin biology and Dr. Douglas

556 Annis to assist with antibody epitope mapping. The authors also thank Ms. Yukun Li to assist

557 with the image analysis using ImageJ. The work was funded by NIH R01 HL129798 (T.J.K.)

558 and NIH U01HL134764 (T.J.K.).

\section{COMPETING INTERESTS}

561 TJK is a consultant for Fujifilm Cellular Dynamics Incorporated.

\section{AUTHOR CONTRIBUTIONS}

564 TJK and JZ conceived the study and wrote the manuscript. JZ designed and carried out the

565 experiments, collected and analyzed the data. RT did most of the cell culture and the qRT-PCR. 
JLC did the RT-PCR. PAL did the FN knockdown construct. YM did the western blot. SPP

contributed funding support and writing/editing the manuscript.

\section{REFERENCES}

[1] D.A.C. Walma, K.M. Yamada, The extracellular matrix in development, Development, 147 (2020). A.G. Soerens, J. Yu, S.P. Palecek, G.E. Lyons, J.A. Thomson, T.J. Herron, J. Jalife, T.J. Kamp, Extracellular matrix promotes highly efficient cardiac differentiation of human pluripotent stem cells: the matrix sandwich method, Circ Res, 111 (2012) 1125-1136. [3] M.A. Nieto, R.Y. Huang, R.A. Jackson, J.P. Thiery, Emt: 2016, Cell, 166 (2016) 21-45. [4] J.W. Lambshead, L. Meagher, C. O'Brien, A.L. Laslett, Defining synthetic surfaces for human pluripotent stem cell culture, Cell Regen, 2 (2013) 7. [5] S. Rodin, L. Antonsson, C. Niaudet, O.E. Simonson, E. Salmela, E.M. Hansson, A. Domogatskaya, Z. Xiao, P. Damdimopoulou, M. Sheikhi, J. Inzunza, A.S. Nilsson, D. Baker, R. Kuiper, Y. Sun, E. Blennow, M. Nordenskjold, K.H. Grinnemo, J. Kere, C. Betsholtz, O. Hovatta, K. Tryggvason, Clonal culturing of human embryonic stem cells on laminin-521/Ecadherin matrix in defined and xeno-free environment, Nature communications, 5 (2014) 3195. [6] L. Yap, J.W. Wang, A. Moreno-Moral, L.Y. Chong, Y. Sun, N. Harmston, X. Wang, S.Y. Chong, K. Vanezis, M.K. Ohman, H. Wei, R. Bunte, S. Gosh, S. Cook, O. Hovatta, D.P.V. de Kleijn, E. Petretto, K. Tryggvason, In Vivo Generation of Post-infarct Human Cardiac Muscle by Laminin-Promoted Cardiovascular Progenitors, Cell reports, 26 (2019) 3231-3245 e3239. [7] J.P. Jung, D. Hu, I.J. Domian, B.M. Ogle, An integrated statistical model for enhanced murine cardiomyocyte differentiation via optimized engagement of 3D extracellular matrices, Scientific reports, 5 (2015) 18705. [8] M.E. Kupfer, W.H. Lin, V. Ravikumar, K. Qiu, L. Wang, L. Gao, D.B. Bhuiyan, M. Lenz, J. Ai, R.R. Mahutga, D. Townsend, J. Zhang, M.C. McAlpine, E.G. Tolkacheva, B.M. Ogle, In Situ Expansion, Differentiation, and Electromechanical Coupling of Human Cardiac Muscle in a 3D Bioprinted, Chambered Organoid, Circ Res, 127 (2020) 207-224.

[9] P.W. Burridge, E. Matsa, P. Shukla, Z.C. Lin, J.M. Churko, A.D. Ebert, F. Lan, S. Diecke, B. Huber, N.M. Mordwinkin, J.R. Plews, O.J. Abilez, B. Cui, J.D. Gold, J.C. Wu, Chemically defined generation of human cardiomyocytes, Nat Methods, 11 (2014) 855-860. [10] J.C. Boucaut, T. Darribere, Fibronectin in early amphibian embryos. Migrating mesodermal cells contact fibronectin established prior to gastrulation, Cell Tissue Res, 234 (1983) 135-145. [11] J.C. Boucaut, T. Darribere, H. Boulekbache, J.P. Thiery, Prevention of gastrulation but not neurulation by antibodies to fibronectin in amphibian embryos, Nature, 307 (1984) 364-367.

604

605 [12] G. Lee, R. Hynes, M. Kirschner, Temporal and spatial regulation of fibronectin in early Xenopus development, Cell, 36 (1984) 729-740.

[13] K.K. Linask, J.W. Lash, Precardiac cell migration: fibronectin localization at mesodermendoderm interface during directional movement, Dev Biol, 114 (1986) 87-101. 
[14] T. Darribere, K.M. Yamada, K.E. Johnson, J.C. Boucaut, The 140-kDa fibronectin receptor complex is required for mesodermal cell adhesion during gastrulation in the amphibian Pleurodeles waltlii, Dev Biol, 126 (1988) 182-194. [15] K.K. Linask, J.W. Lash, A role for fibronectin in the migration of avian precardiac cells. I. Dose-dependent effects of fibronectin antibody, Dev Biol, 129 (1988) 315-323. [16] K.K. Linask, J.W. Lash, A role for fibronectin in the migration of avian precardiac cells. II. Rotation of the heart-forming region during different stages and its effects, Dev Biol, 129 (1988) 324-329. fibrillar extracellular matrix is required for normal Rana pipiens gastrulation, J Exp Zool, 265 (1993) 40-53.

[18] H.R. Suzuki, M. Solursh, H.S. Baldwin, Relationship between fibronectin expression during gastrulation and heart formation in the rat embryo, Dev Dyn, 204 (1995) 259-277. [19] J.C. Boucaut, L. Clavilier, T. Darribere, M. Delarue, J.F. Riou, D.L. Shi, What mechanisms drive cell migration and cell interactions in Pleurodeles?, Int J Dev Biol, 40 (1996) 675-683. [20] J.P. Thiery, J.P. Sleeman, Complex networks orchestrate epithelial-mesenchymal transitions, Nat Rev Mol Cell Biol, 7 (2006) 131-142.

[21] J. Lim, J.P. Thiery, Epithelial-mesenchymal transitions: insights from development, Development, 139 (2012) 3471-3486.

[22] M. Leptin, B. Grunewald, Cell shape changes during gastrulation in Drosophila, Development, 110 (1990) 73-84.

[23] M.A. Nieto, M.G. Sargent, D.G. Wilkinson, J. Cooke, Control of cell behavior during vertebrate development by Slug, a zinc finger gene, Science, 264 (1994) 835-839.

[24] J.P. Thiery, H. Acloque, R.Y. Huang, M.A. Nieto, Epithelial-mesenchymal transitions in development and disease, Cell, 139 (2009) 871-890. [25] M.A. Nieto, The snail superfamily of zinc-finger transcription factors, Nat Rev Mol Cell Biol, 3 (2002) 155-166.

[26] A. Barrallo-Gimeno, M.A. Nieto, The Snail genes as inducers of cell movement and survival: implications in development and cancer, Development, 132 (2005) 3151-3161. [27] M. Bachmann, S. Kukkurainen, V.P. Hytonen, B. Wehrle-Haller, Cell Adhesion by Integrins, Physiol Rev, 99 (2019) 1655-1699.

[28] M. Bharadwaj, N. Strohmeyer, G.P. Colo, J. Helenius, N. Beerenwinkel, H.B. Schiller, R. Fassler, D.J. Muller, alphaV-class integrins exert dual roles on alpha5beta1 integrins to 640 [29] G.E. Hannigan, C. Leung-Hagesteijn, L. Fitz-Gibbon, M.G. Coppolino, G. Radeva, J. 641 Filmus, J.C. Bell, S. Dedhar, Regulation of cell adhesion and anchorage-dependent growth by a new beta 1-integrin-linked protein kinase, Nature, 379 (1996) 91-96.

[30] M. Delcommenne, C. Tan, V. Gray, L. Rue, J. Woodgett, S. Dedhar, Phosphoinositide-3$\mathrm{OH}$ kinase-dependent regulation of glycogen synthase kinase 3 and protein kinase B/AKT by the integrin-linked kinase, Proc Natl Acad Sci U S A, 95 (1998) 11211-11216. [31] A. Oloumi, T. McPhee, S. Dedhar, Regulation of E-cadherin expression and betacatenin/Tcf transcriptional activity by the integrin-linked kinase, Biochim Biophys Acta, 1691 (2004) 1-15.

[32] X. Lian, C. Hsiao, G. Wilson, K. Zhu, L.B. Hazeltine, S.M. Azarin, K.K. Raval, J. Zhang, T.J. Kamp, S.P. Palecek, Robust cardiomyocyte differentiation from human pluripotent stem 
cells via temporal modulation of canonical Wnt signaling, Proc Natl Acad Sci U S A, 109 (2012) E1848-1857. [33] X. Lian, J. Zhang, S.M. Azarin, K. Zhu, L.B. Hazeltine, X. Bao, C. Hsiao, T.J. Kamp, S.P. Palecek, Directed cardiomyocyte differentiation from human pluripotent stem cells by modulating Wnt/beta-catenin signaling under fully defined conditions, Nat Protoc, 8 (2013) 162175. [34] L. Yang, M.H. Soonpaa, E.D. Adler, T.K. Roepke, S.J. Kattman, M. Kennedy, E. Henckaerts, K. Bonham, G.W. Abbott, R.M. Linden, L.J. Field, G.M. Keller, Human cardiovascular progenitor cells develop from a KDR+ embryonic-stem-cell-derived population, Nature, 453 (2008) 524-528. [35] S.J. Kattman, A.D. Witty, M. Gagliardi, N.C. Dubois, M. Niapour, A. Hotta, J. Ellis, G. Keller, Stage-specific optimization of activin/nodal and BMP signaling promotes cardiac differentiation of mouse and human pluripotent stem cell lines, Cell Stem Cell, 8 (2011) 228240.

[36] N.C. Dubois, A.M. Craft, P. Sharma, D.A. Elliott, E.G. Stanley, A.G. Elefanty, A. Gramolini, G. Keller, SIRPA is a specific cell-surface marker for isolating cardiomyocytes derived from human pluripotent stem cells, Nat Biotechnol, 29 (2011) 1011-1018.

[37] F.L. Conlon, K.M. Lyons, N. Takaesu, K.S. Barth, A. Kispert, B. Herrmann, E.J. Robertson, A primary requirement for nodal in the formation and maintenance of the primitive streak in the mouse, Development, 120 (1994) 1919-1928.

[38] J. Lough, M. Barron, M. Brogley, Y. Sugi, D.L. Bolender, X. Zhu, Combined BMP-2 and FGF-4, but neither factor alone, induces cardiogenesis in non-precardiac embryonic mesoderm, Dev Biol, 178 (1996) 198-202.

[39] T. Mima, H. Ueno, D.A. Fischman, L.T. Williams, T. Mikawa, Fibroblast growth factor receptor is required for in vivo cardiac myocyte proliferation at early embryonic stages of heart development, Proc Natl Acad Sci U S A, 92 (1995) 467-471.

[40] G. Winnier, M. Blessing, P.A. Labosky, B.L. Hogan, Bone morphogenetic protein-4 is required for mesoderm formation and patterning in the mouse, Genes Dev, 9 (1995) 2105-2116. [41] M.A. Laflamme, K.Y. Chen, A.V. Naumova, V. Muskheli, J.A. Fugate, S.K. Dupras, H. Reinecke, C. Xu, M. Hassanipour, S. Police, C. O'Sullivan, L. Collins, Y. Chen, E. Minami, E.A. Gill, S. Ueno, C. Yuan, J. Gold, C.E. Murry, Cardiomyocytes derived from human embryonic stem cells in pro-survival factors enhance function of infarcted rat hearts, Nat Biotechnol, 25 (2007) 1015-1024.

[42] J.L. Duband, J.P. Thiery, Appearance and distribution of fibronectin during chick embryo gastrulation and neurulation, Dev Biol, 94 (1982) 337-350.

[43] E.L. George, E.N. Georges-Labouesse, R.S. Patel-King, H. Rayburn, R.O. Hynes, Defects in mesoderm, neural tube and vascular development in mouse embryos lacking fibronectin, Development, 119 (1993) 1079-1091. Biol, 87 (1980) 309-313.

694 J.A. McDonald, S. Dedhar, Integrin-linked protein kinase regulates fibronectin matrix assembly, E-cadherin expression, and tumorigenicity, J Biol Chem, 273 (1998) 528-536. 
697 [47] A.J. Engler, S. Sen, H.L. Sweeney, D.E. Discher, Matrix elasticity directs stem cell lineage 698 specification, Cell, 126 (2006) 677-689.

699 [48] T. Rozario, D.W. DeSimone, The extracellular matrix in development and morphogenesis: a 700 dynamic view, Dev Biol, 341 (2010) 126-140.

701 [49] A. Laperle, C. Hsiao, M. Lampe, J. Mortier, K. Saha, S.P. Palecek, K.S. Masters, alpha-5 702 Laminin Synthesized by Human Pluripotent Stem Cells Promotes Self-Renewal, Stem Cell

703 Reports, 5 (2015) 195-206.

704 [50] J. Zhang, G.F. Wilson, A.G. Soerens, C.H. Koonce, J. Yu, S.P. Palecek, J.A. Thomson, T.J. 705 Kamp, Functional cardiomyocytes derived from human induced pluripotent stem cells, Circ Res, $706104(2009)$ e 30-41. 
Fig. 1A

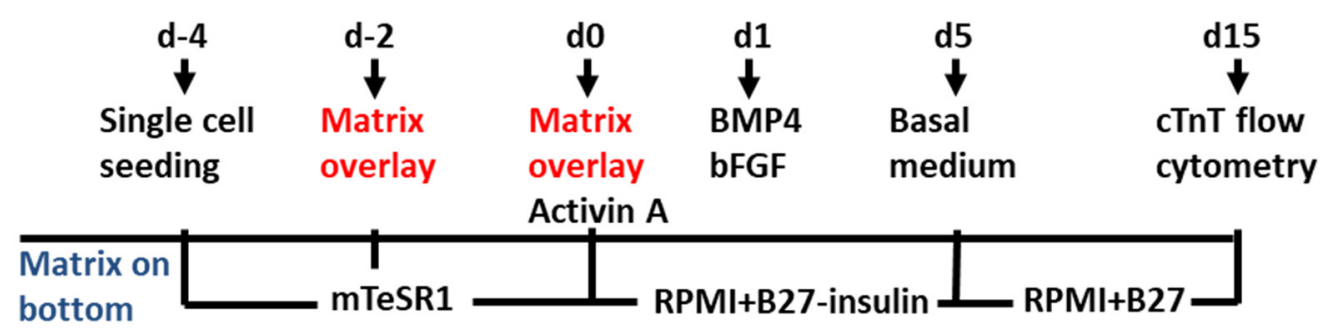

Fig. 1B

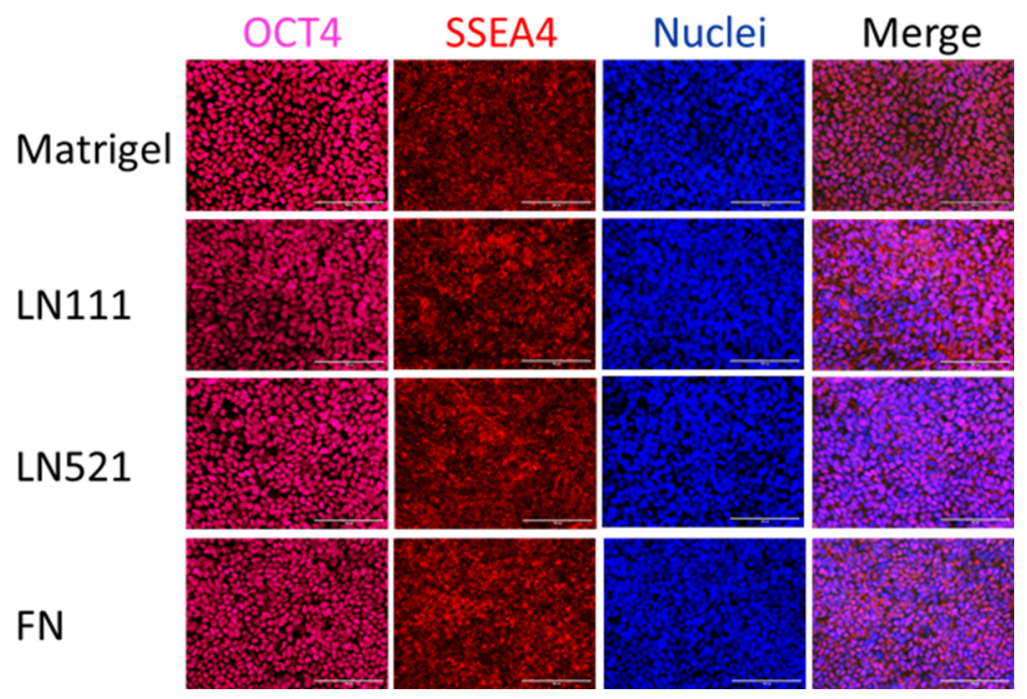

Fig. 1C

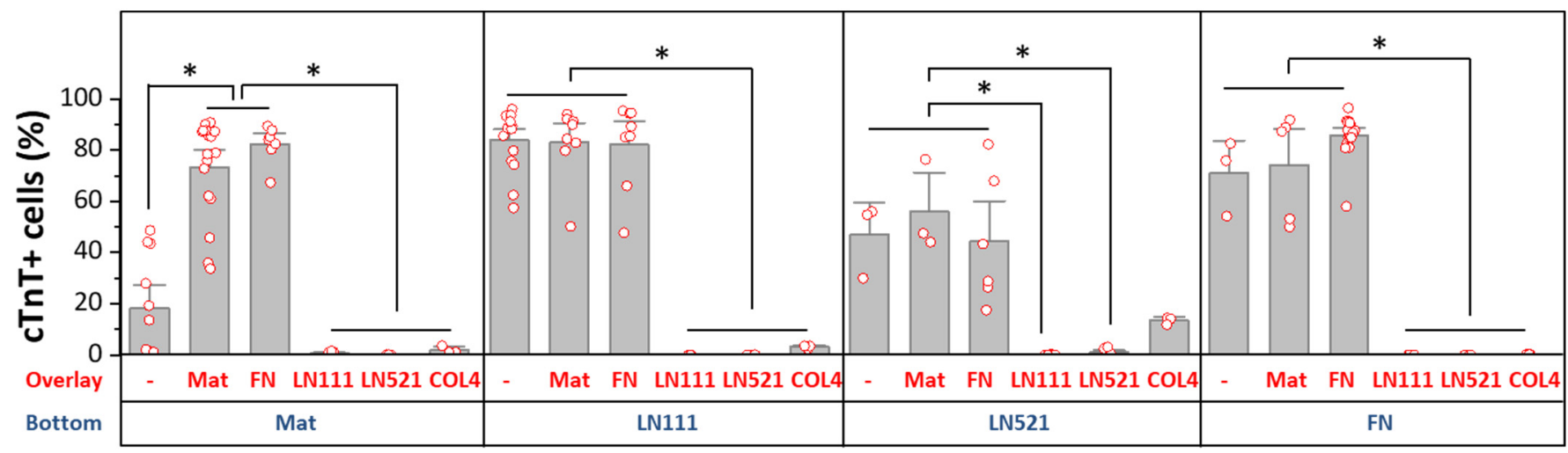

Fig. 1. Defined ECM proteins support hPSCs adhesion, growth and cardiac differentiation using the matrix sandwich protocol. (A) Schematic method of the matrix sandwich protocol. Defined ECM proteins are tested as coating matrix and overlay matrix in RED. (B) Fluorescence images of DF19-9-11T iPSCs growing on the ECM of Matrigel, LN111, LN521 and FN as confluent monolayer and immuno-labeled with antibodies against OCT4 and SSEA4. Scale bar is $100 \mu \mathrm{m}$. (C) $\mathrm{cTnT}^{+}$cells measured by flow cytometry at 15 days of differentiation of DF19-9$11 \mathrm{~T}$ iPSCs on different ECM proteins as substrate (bottom) and overlay (overlay). $\mathrm{N} \geq 3$ biological replicates. Error bars represent SEM. ${ }^{*} \mathrm{P}<0.05$, one-way ANOVA with post-hoc Bonferroni and Tukey test. 
Fig. 2A

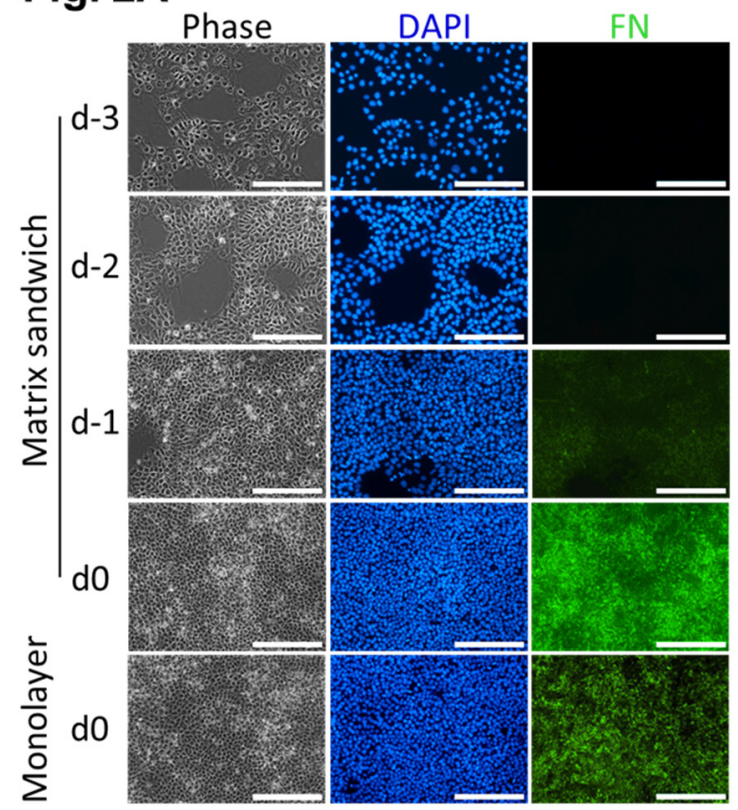

Fig. 2D

Fig. 2C

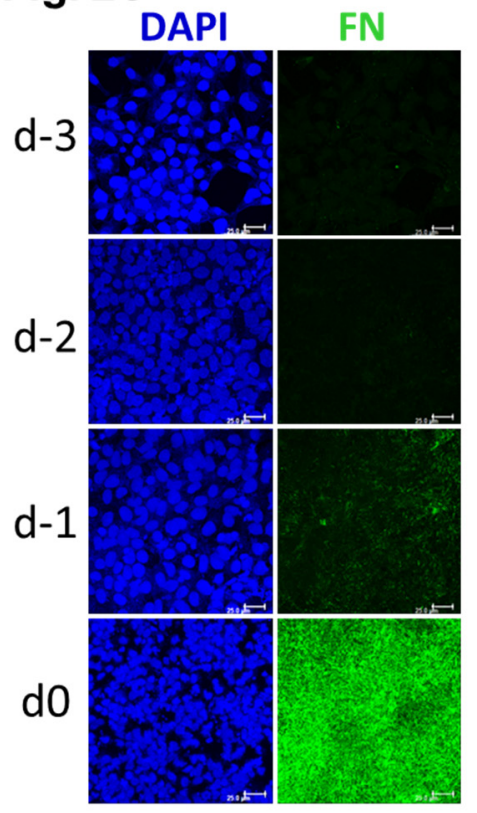

Fig. 2B

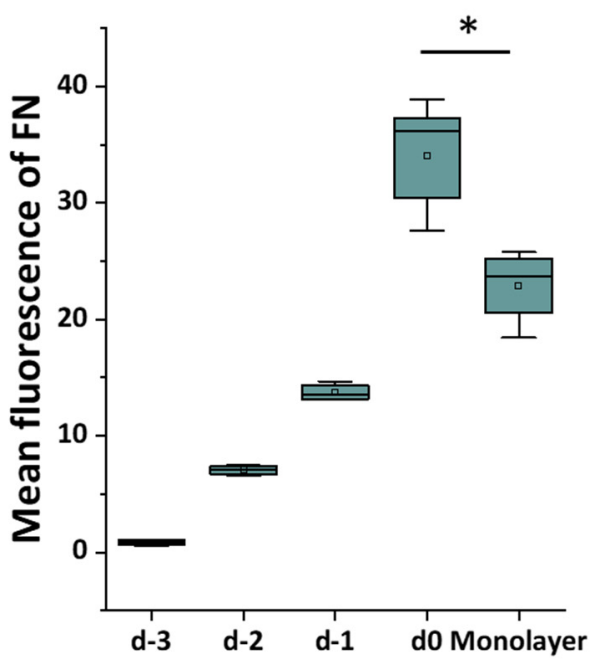

Fig. 2. Production of endogenous $\mathrm{FN}$ in the hPSCmatrix sandwich and LN111 culture. (A) Phase contrast and fluorescence images of the matrix sandwich culture of DF19-9$11 \mathrm{~T}$ iPSCs growing for 4 days and immunolabeled with fibronectin antibody, compared with the monolayer culture. Scale bar is 200 $\mu \mathrm{m}$. (B) Quantitative analysis of the FN fluorescence in A by Image $J$. $N \geq 3$ replicates. The box plots summarize the biological replicates with the box enclosing from first to third quartile and middle square indicating mean and line in box indicating

median. ${ }^{*} \mathrm{P}<0.05$, one-way ANOVA with post-hoc Bonferroni and Tukey test. (C) Maximum projection view of the confocal z-scan of DF19-9-11T iPSCs growing for 4 days on LN111 coated surface immunolabeled with fibronectin antibody. Scale bar is $25 \mu \mathrm{m}$. (D) The maximum projection view and side view of the confocal z-scan of DF19-9-11T iPSCs growing for 4 days on LN111 coated surface without matrix overlay and immunolabeled with the fibronectin antibody. The multiplayer culture and FN production are similar as in Matrigel/Matrigel and FN/FN matrix sandwich culture. Scale bar is $25 \mu \mathrm{m}$. 
Fig. 3A

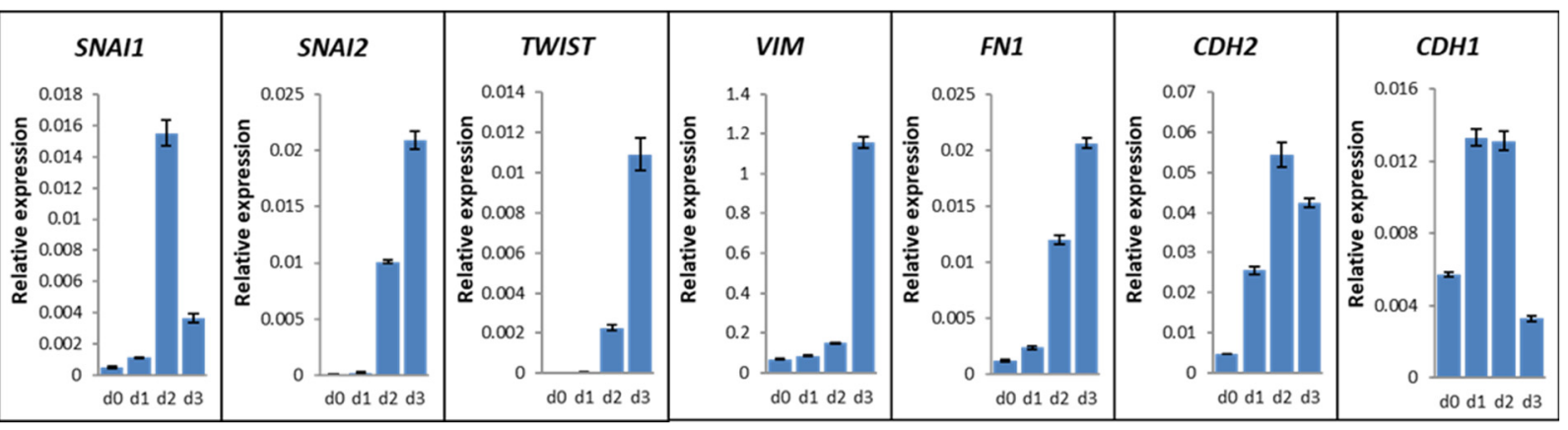

Fig. 3B
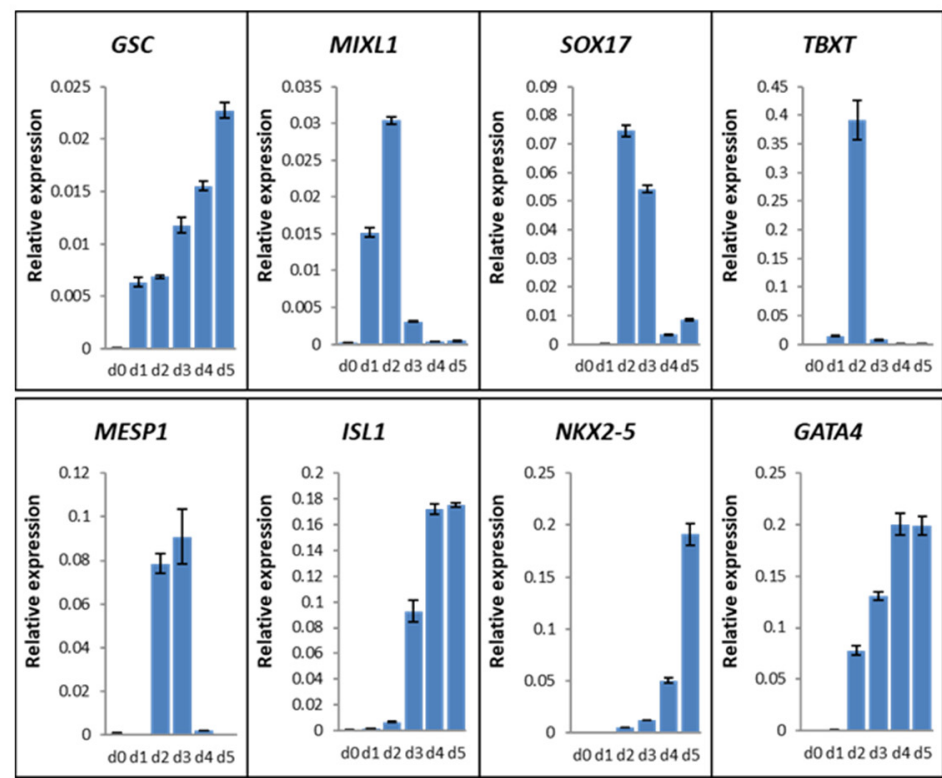

Fig. 3C

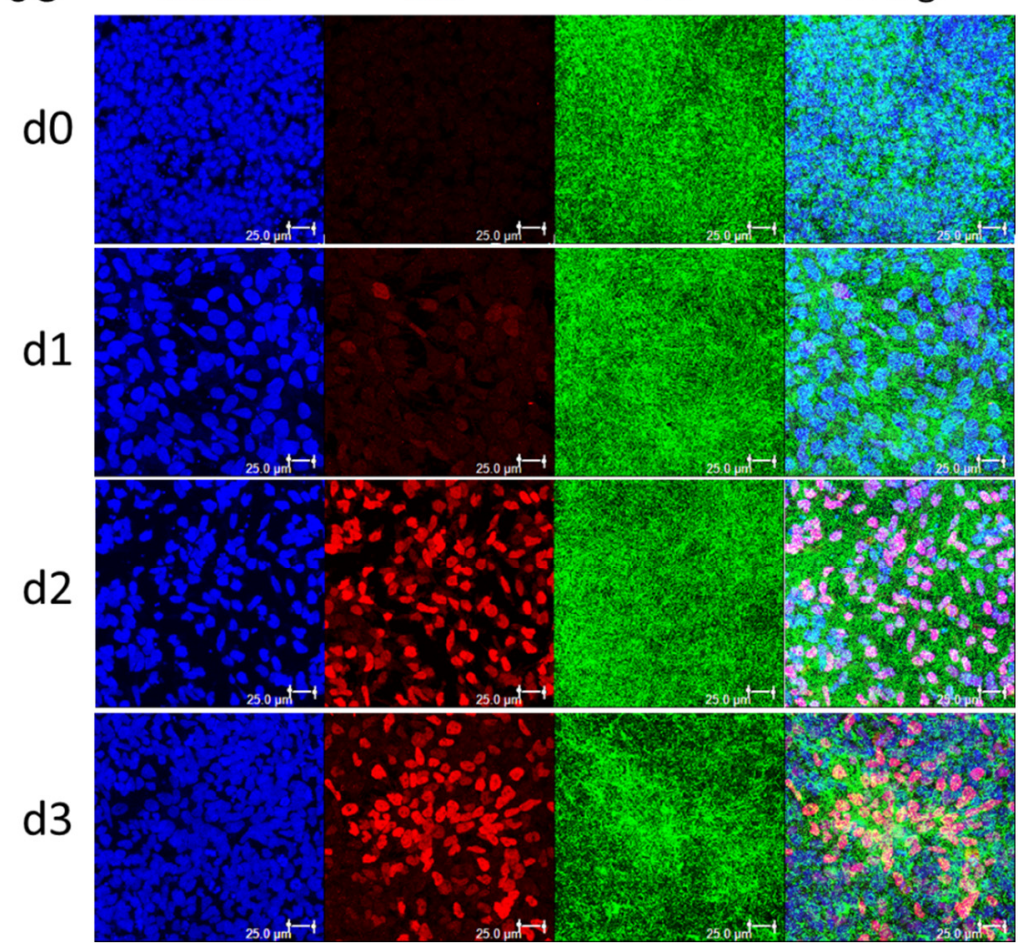

Fig. 3. Expression of EMT and precardiac mesoderm markers in the cardiac differentiation of hPSCs cultured on LN111 substrate by Activin A/BMP4/bFGF signaling. (A) qRT-PCR for gene expression of EMT markers at day 0-3 of cardiac differentiation. (B) qRT-PCR for gene expression of precardiac mesoderm markers at day 0-5 of cardiac differentiation. $\mathrm{N}=3$ technical replicates for each point. (C) Maximum projection view of the confocal z-scan of DF19-9$11 \mathrm{~T}$ iPSCs at day $0-3$ of the cardiac differentiation colabeled with antibodies against Brachyury (BRY) and Fibronectin (FN). Scale bar is $25 \mu \mathrm{m}$. Error bars represent SEM. 
Fig. 4A

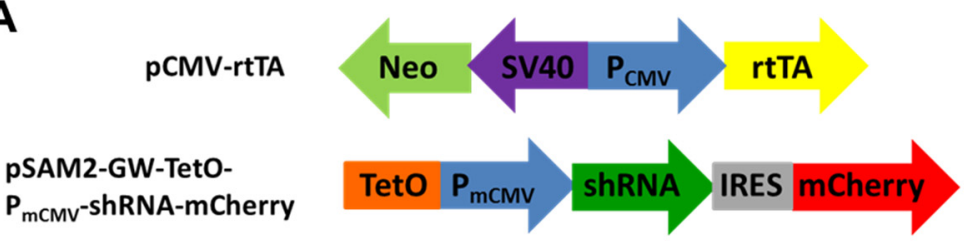

Fig. 4B

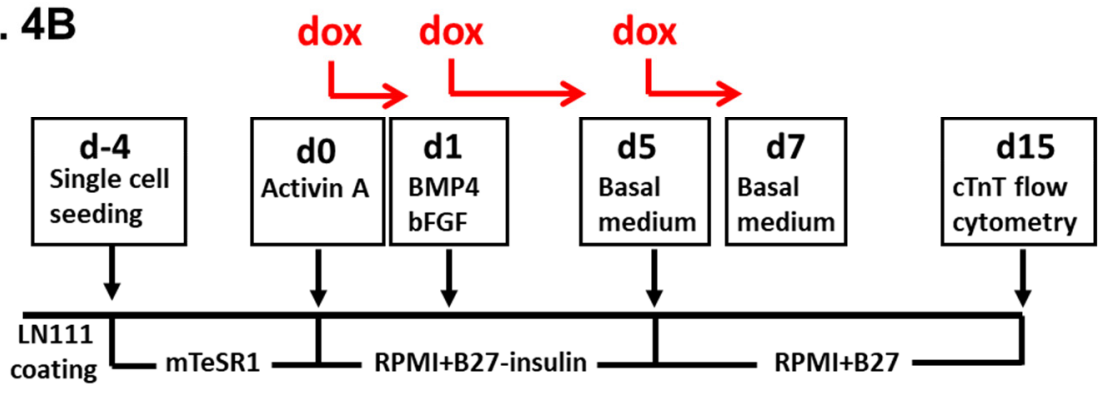

Fig. 4C

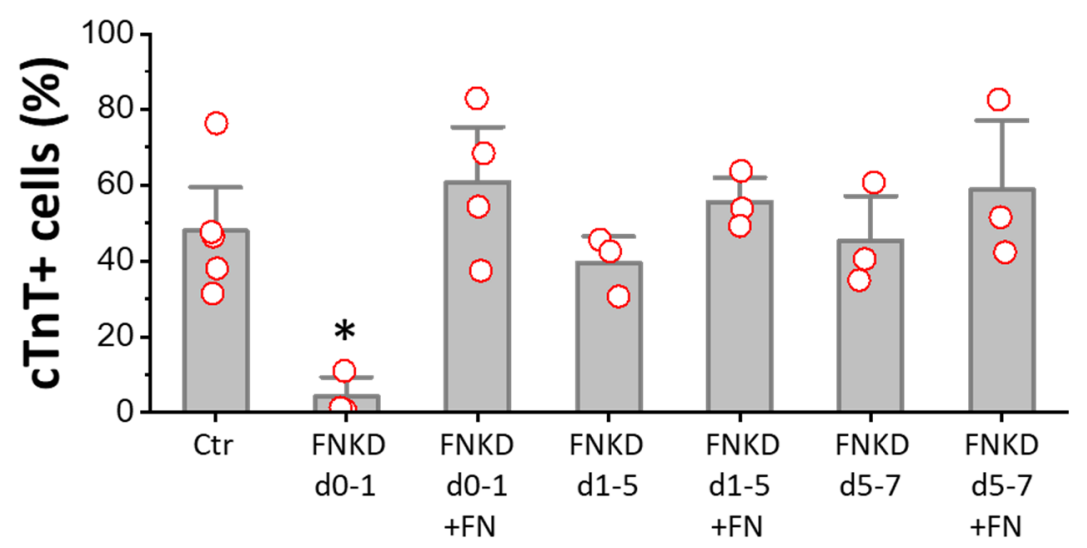

Fig. 4. FN is essential for precardiac mesoderm formation in the cardiac differentiation of hPSCs. (A) Schematic of the inducible shRNA construct for $F N 1$ knockdown. (B) Schematic method of FN knockdown at differentiation stages of day 0-1, 1-5 and day 5-7 in the cardiac differentiation protocol. (C) $\mathrm{cTnT}^{+}$cells measured by flow cytometry at 15 days of differentiation of the $\mathrm{H} 1 F N 1$ knockdown clone using the protocol in Fig. 4B. N $\geq 3$ biological replicates. Error bars represent SEM. ${ }^{*} \mathrm{P}<0.05$, one-way ANOVA with post-hoc Bonferroni and Tukey test. FNKD indicates FN knockdown by dox induction. +FN indicates exogenous FN added. 
Fig. 5A

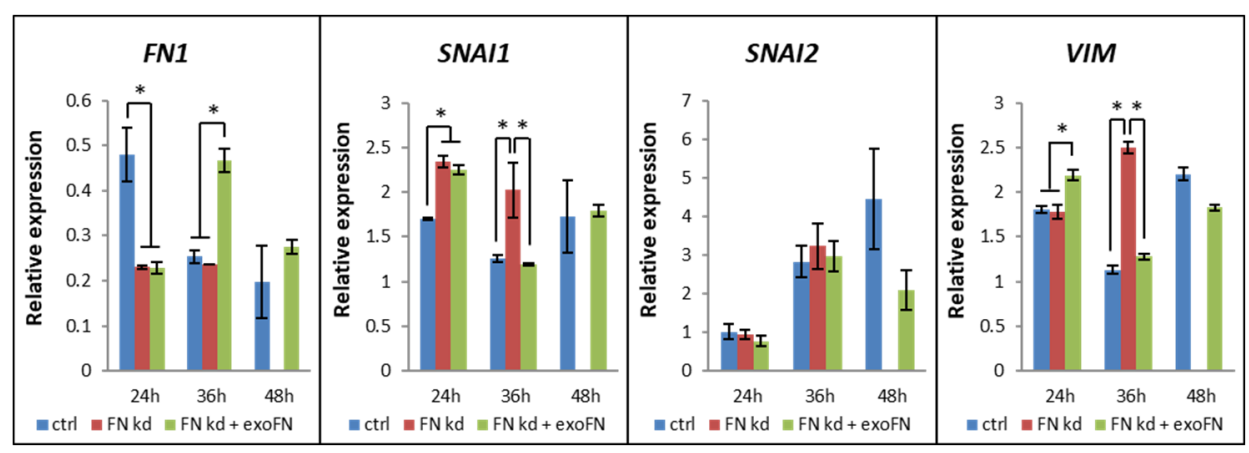

Fig. 5B

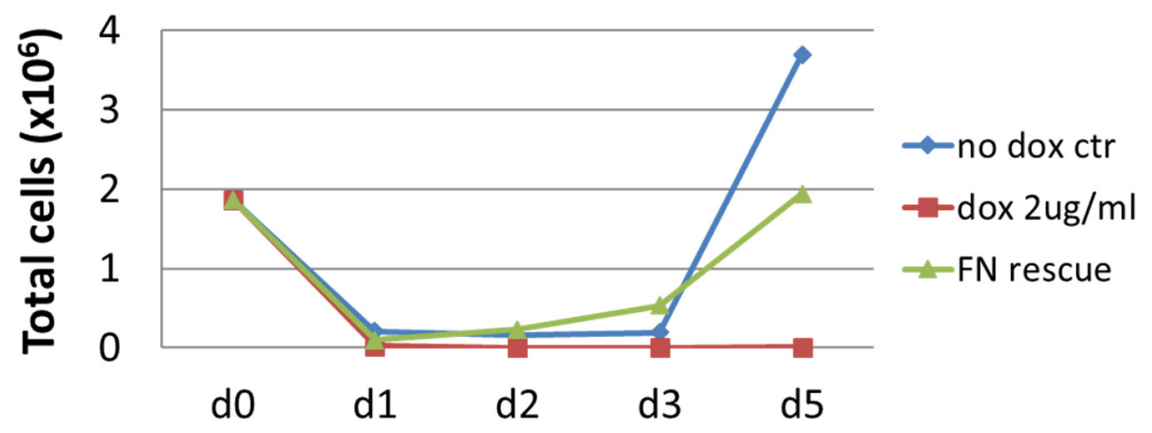

Fig. 5C

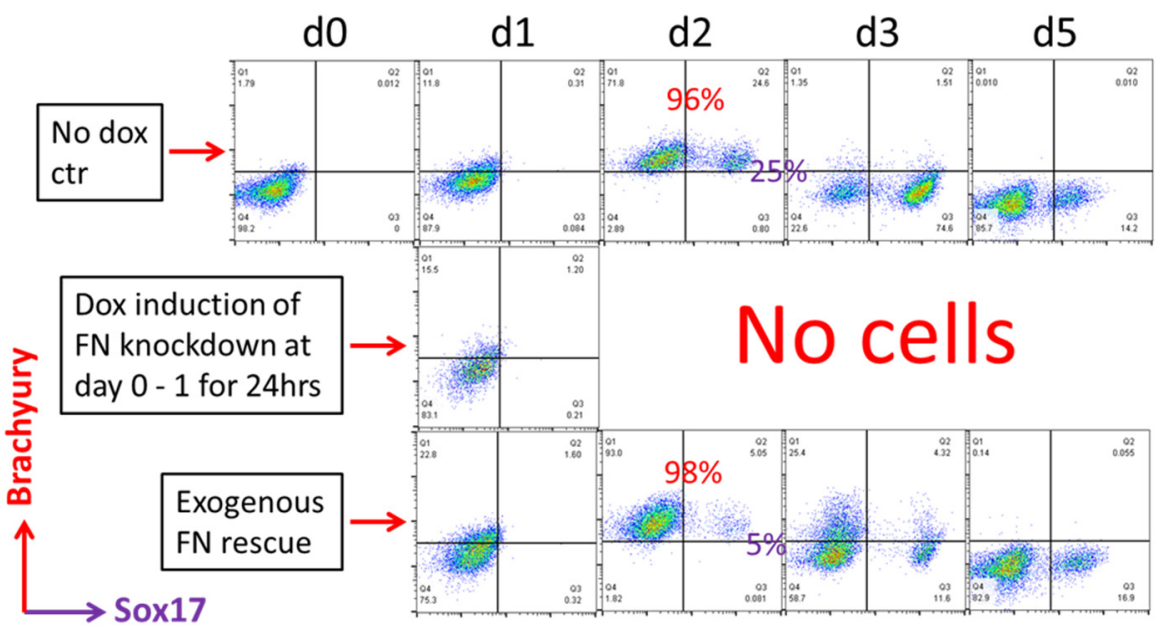

Fig. 5. Knockdown FN at the precardiac mesoderm stage in the Activin A/BMP4/bFGF directed cardiac differentiation results in loss of Brachyury ${ }^{+}$cells, exogenous FN can rescue it. (A) qRT-PCR for gene expression of EMT markers for the H1 FN knockdown clone in the cardiac differentiation time course of $0-48 \mathrm{~h}$ at the no dox control, dox induction at day $0-1$ and dox induction at day $0-1$ with adding exogenous FN conditons. (B) Total cell number of the H1 FN knockdown clone in the time course of day 0-5 in the cardiac differentiation at the no dox control, dox induction at day 0-1 and dox induction at day 0-1 with adding exogenous FN conditions. (C) Flow cytometry of co-labeling the cells shown on (B) with Brachyury and Sox 17 antibodies. Error bars represent SEM. ${ }^{*} \mathrm{P}<0.05$, one-way ANOVA with post-hoc Bonferroni and Tukey test. 
Fig. 6A

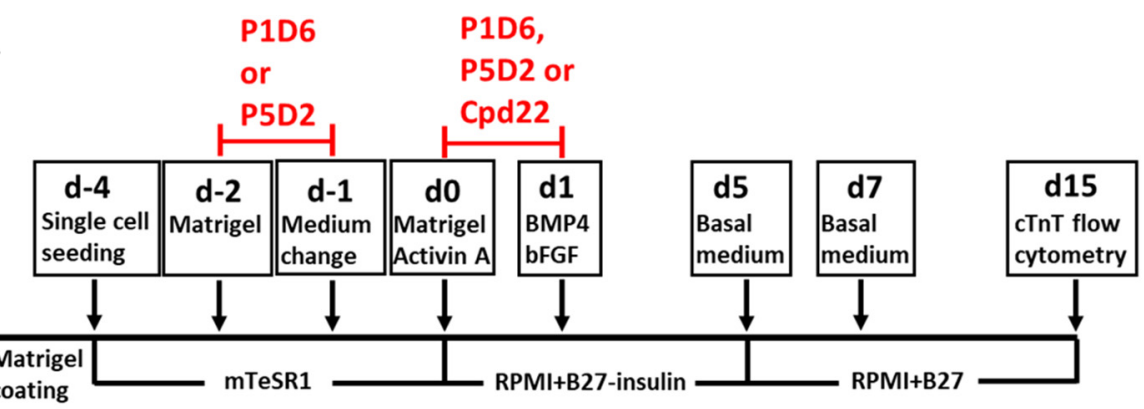

Fig. 6B

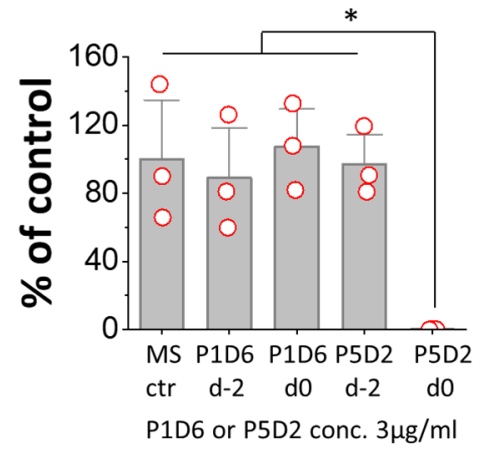

Fig. 6E

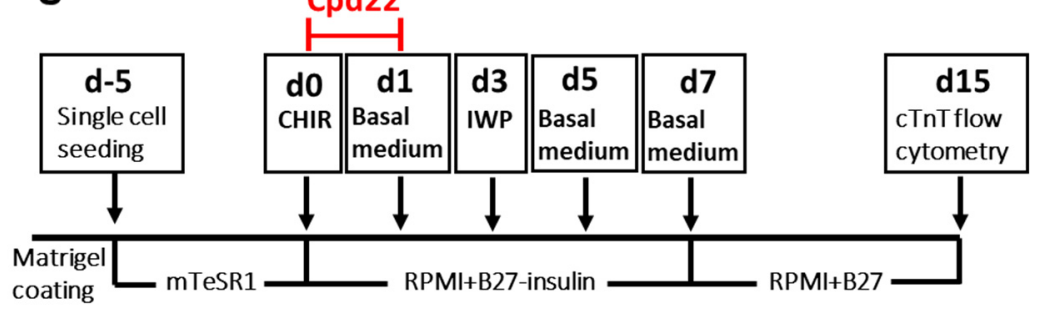

Fig. 6C

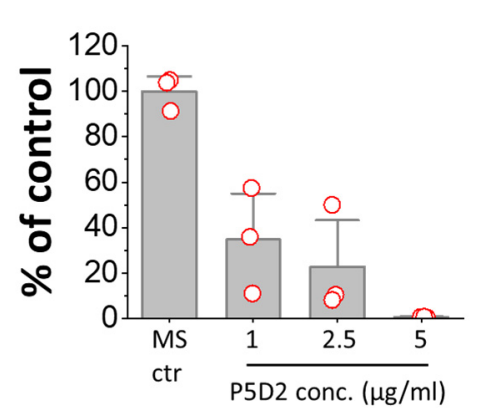

Fig. 6D

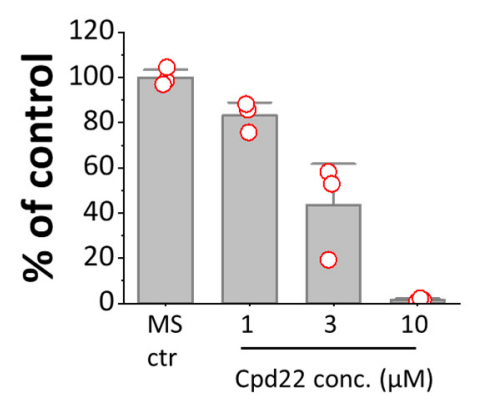

Fig. $6 \mathrm{~F}$

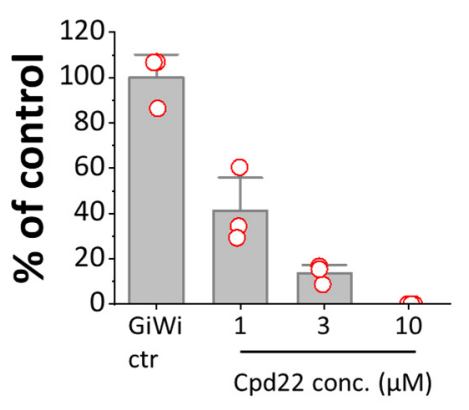

Fig. 6. Cardiac differentiation is blocked by integrin $\beta 1$ antibody and ILK inhibitor when added at day 0 in the matrix sandwich and GiWi protocols. (A) Schematic for testing monoclonal antibodies to block integrins $\alpha 5$ and $\beta 1$ (P1D6 and P5D2, respectively) and inhibition of ILK by small molecule inhibitor Cpd22 in the matrix sandwich (MS) protocol. (B) $\mathrm{cTnT}^{+}$cells measured by flow cytometry at 15 days differentiation when anti-human integrin $\alpha 5$ (P1D6) or $\beta 1$ (P5D2) antibody was added at day -2 or day 0 at $3 \mu \mathrm{g} / \mathrm{ml}$ in the matrix sandwich protocol as shown in $\mathrm{A}$. (C) $\mathrm{cTnT}^{+}$cells measured by flow cytometry at 15 days differentiation when antihuman integrin $\beta 1$ antibody (P5D2) was added at day 0 at concentrations of 0 (Ctr), 1, 2.5 and 5 $\mu \mathrm{g} / \mathrm{ml}$ in the matrix sandwich protocol as shown in A. (D) $\mathrm{cTnT}^{+}$cells measured by flow cytometry at 15 days differentiation when $\mathrm{Cpd} 22$ was added at day 0 at concentrations of 0 (Ctr), 1,3 and $10 \mu \mathrm{M}$ in the matrix sandwich protocol as shown in A. (E) Schematic for testing ILK inhibitor $\mathrm{Cpd} 22$ in the GiWi protocol. (F) $\mathrm{cTnT}^{+}$cells measured by flow cytometry at 15 days differentiation when $\mathrm{Cpd} 22$ was added at day 0 at concentrations of 0 (Ctr), 1,3 and $10 \mu \mathrm{M}$ in the GiWi protocol as shown in $\mathrm{E}$. The $\%$ of $\mathrm{cTnT}^{+}$cells in each group was normalized to the control and presented as \% of control. $\mathrm{N}=3$ biological replicates. Data are from DF19-9-11T iPSC line. Error bars represent SEM. $* \mathrm{P}<0.05$, one-way ANOVA with post-hoc Bonferroni and Tukey test. 
bioRxiv preprint doi: https://doi.org/10.1101/2021.04.09.439173; this version posted April 11, 2021. The copyright holder for this preprint (which

was not certified by peer review) is the author/funder, who has granted bioRxiv a license to display the preprint in perpetuity. It is made available under aCC-BY 4.0 International license.

\section{Cardiac Differentiation of Human Pluripotent Stem Cells Using Defined Extracellular Matrix Proteins Reveals Essential Role of Fibronectin}

Zhang et al. 


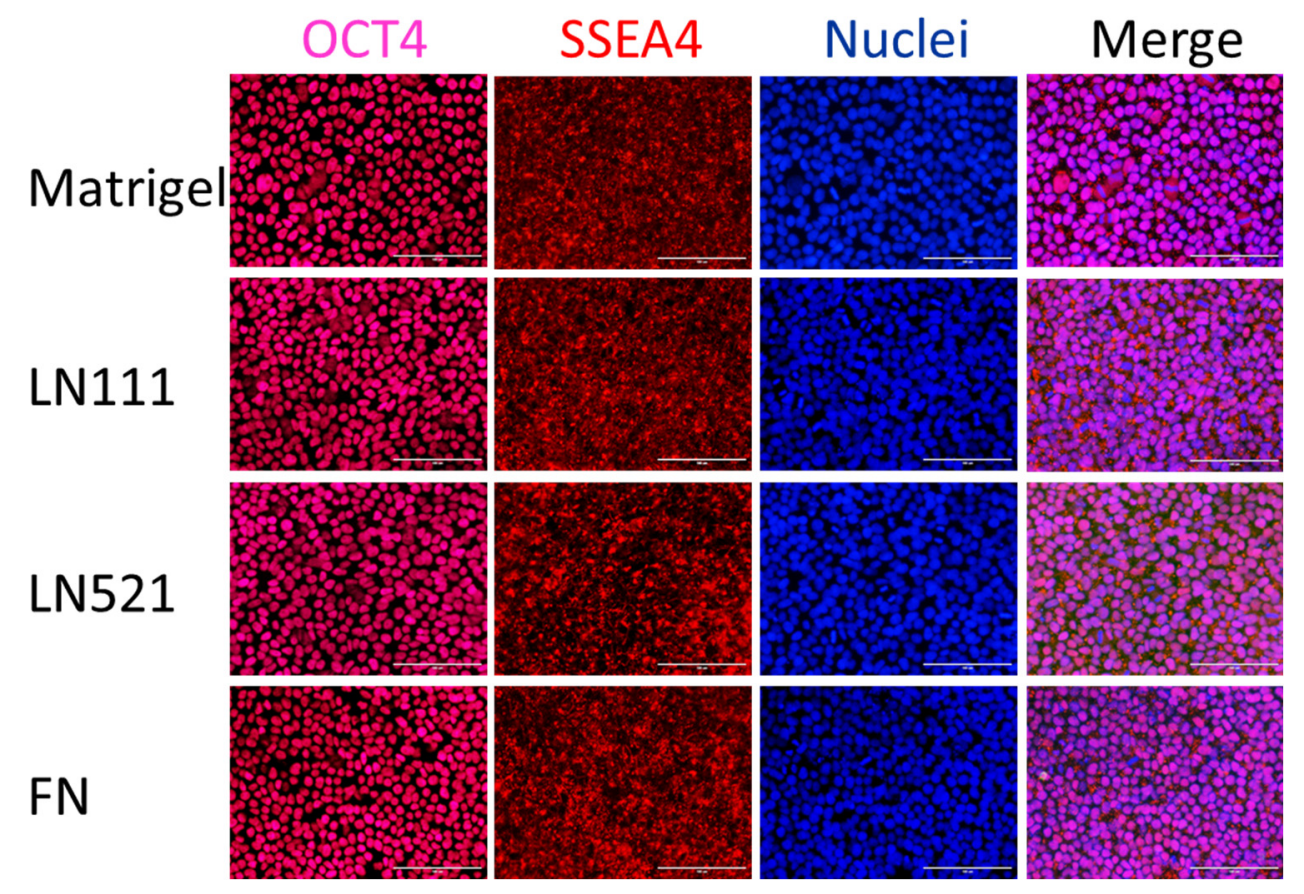

Supplementary Fig. S1 Fluorescence images of H1 ESCs growing on the ECM of Matrigel, LN111, LN521 and FN as confluent monolayer and immunolabeled with antibodies against OCT4 and SSEA4. Scale bar is $100 \mu \mathrm{m}$.
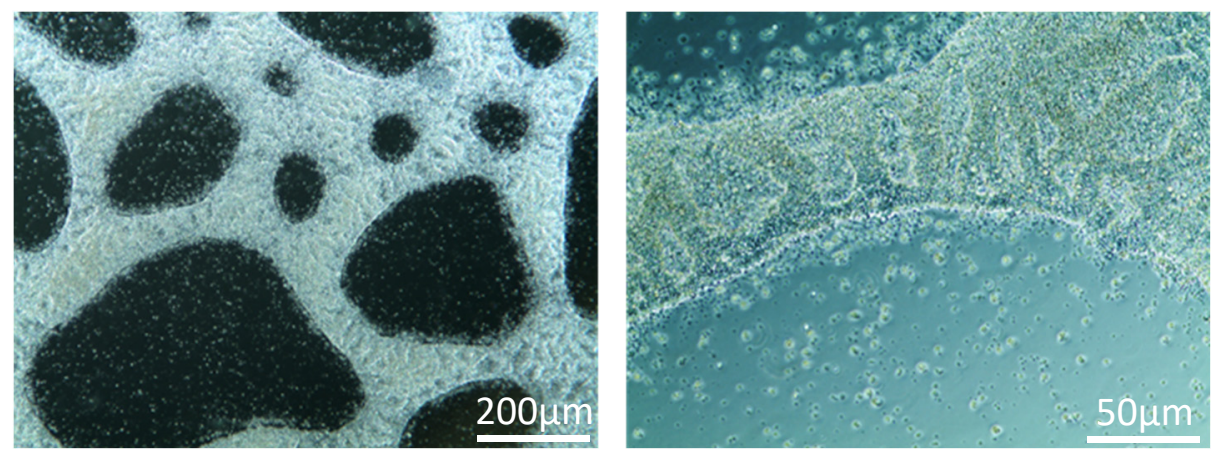

Supplementary Fig. S2 Morphology of DF19-9-11 iPSCs growing on human collagen IV coated surface. The iPSCs growing for 5 days on collagen IV coated surface do not form confluent monolayer. 


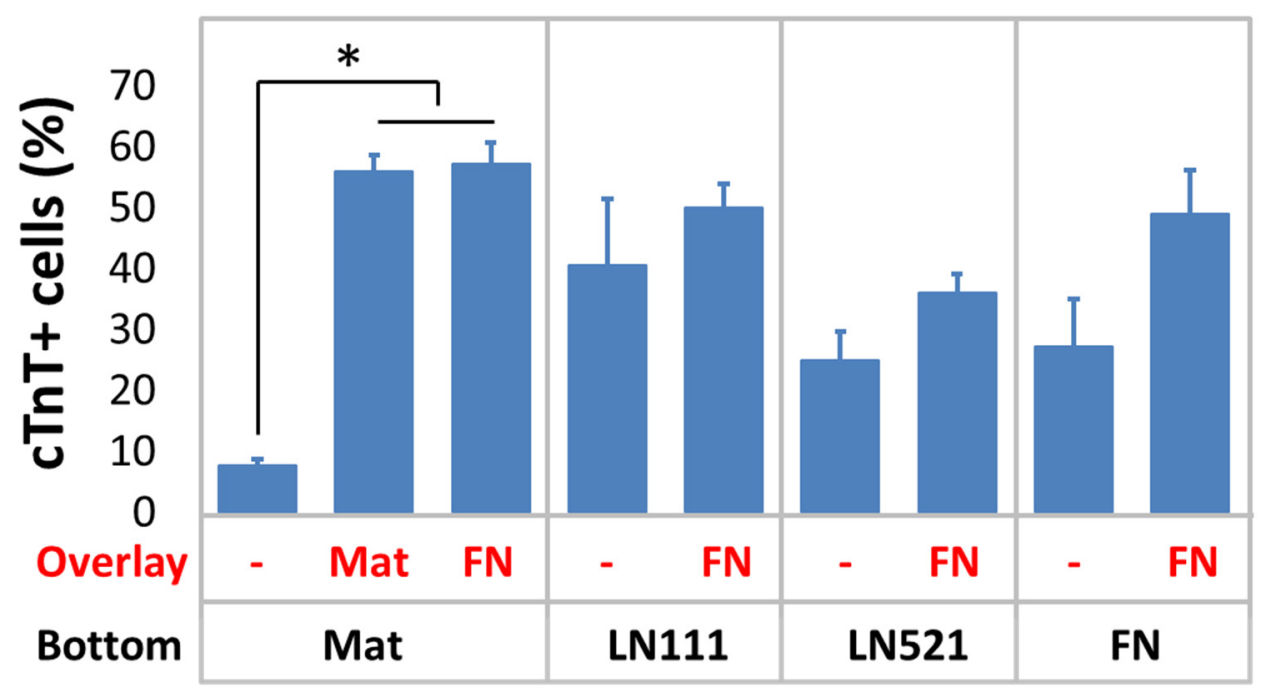

Supplementary Fig. S3 cTnT $^{+}$cells measured by flow cytometry at 15 days of differentiation of H1 ESCs on different ECM proteins as substrate (bottom) and overlay (overlay) using the matrix sandwich protocol. $\mathrm{N} \geq 3$ biological replicates. ${ }^{*} \mathrm{P}<0.05$, one-way ANOVA with post-hoc Bonferroni and Tukey test.

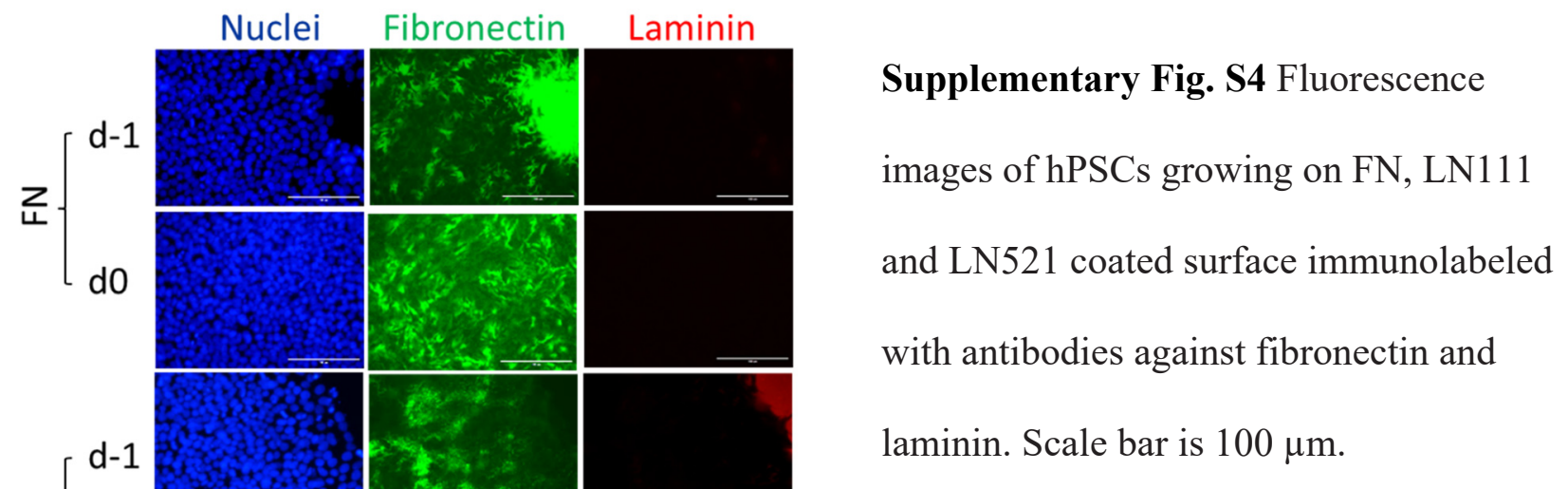



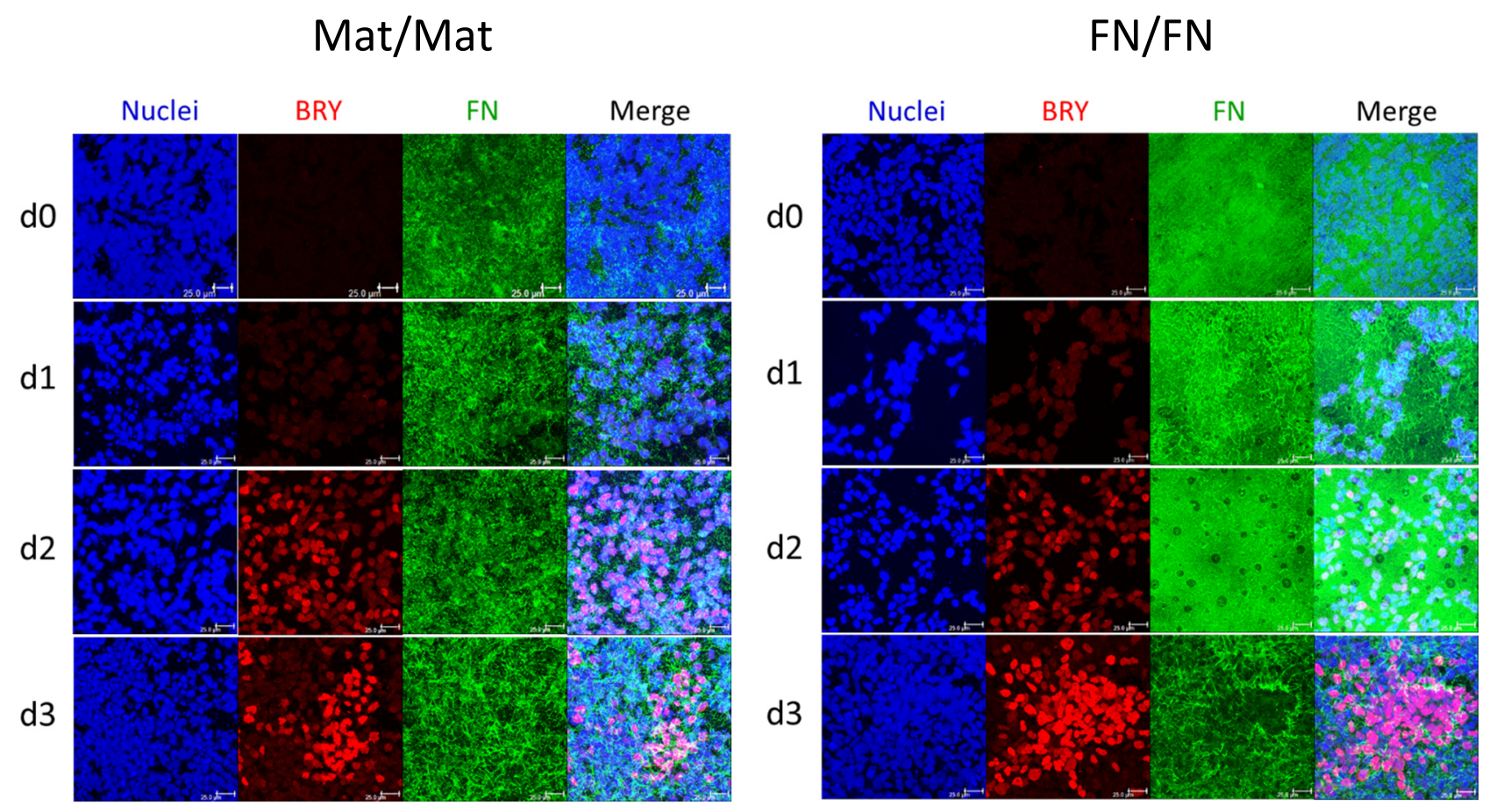

Supplementary Fig. S5 Maximum projection view of the confocal z-scan of DF19-9-11T iPSCs at day 0-3 of the cardiac differentiation using the Matrigel/Matrigel (Mat/Mat) and FN/FN matrix sandwich protocol. The cells were co-labeled with antibodies against brachyury (BRY) and fibronectin (FN). Scale bar is $25 \mu \mathrm{m}$.

\section{Sigma oligo-1}

Xho1 CCGGTGCAGCACAACTTCGAATTATCTCGAGATAATTCGAAGTTGTGCTGCATTTTTG

Age1

FN1 KD1 F - 5' ACCGGTGCAGCACAACTTCGAATTATCTCGAGATAATTCGAAGTTGTGCTGCATTTTTGGAATTCC 3' FN1 KD1 R - 5' GGAATTCCAAAAATGCAGCACAACTTCGAATTATCTCGAGATAATTCGAAGTTGTGCTGCACCGGT 3'

\section{Sigma oligo-2}

CCGGAGATTTGGTTTGGGATCAATACTCGAGTATTGATCCCAAACCAAATCTTTTTTG

FN1 KD2 F - 5' ACCGGTAGATTTGGTTTGGGATCAATACTCGAGTATTGATCCCAAACCAAATCTTTTTTGGAATTCC 3' FN1 KD2 R - 5' GGAATTCCAAAAAAGATTTGGTTTGGGATCAATACTCGAGTATTGATCCCAAACCAAATCTACCGGT 3'

Supplementary Fig. S6 The FN1 shRNA oligos. Yellow, CDS where shRNA binds; Green, palindrome. Oligo-1 binds in exon 8 (95\% KD); Oligo-2 binds in 3' UTR (93\% KD). 
A

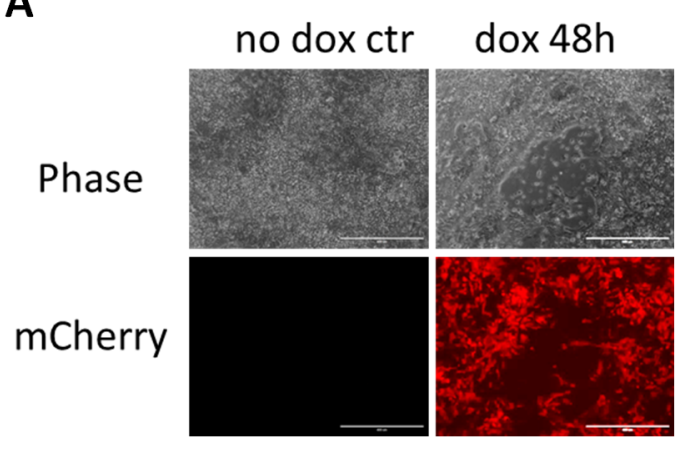

B

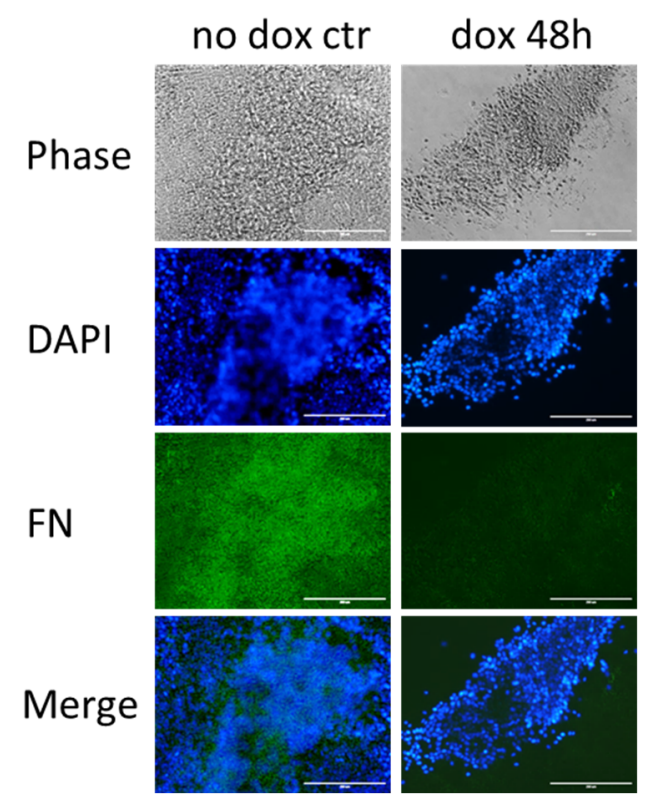

C
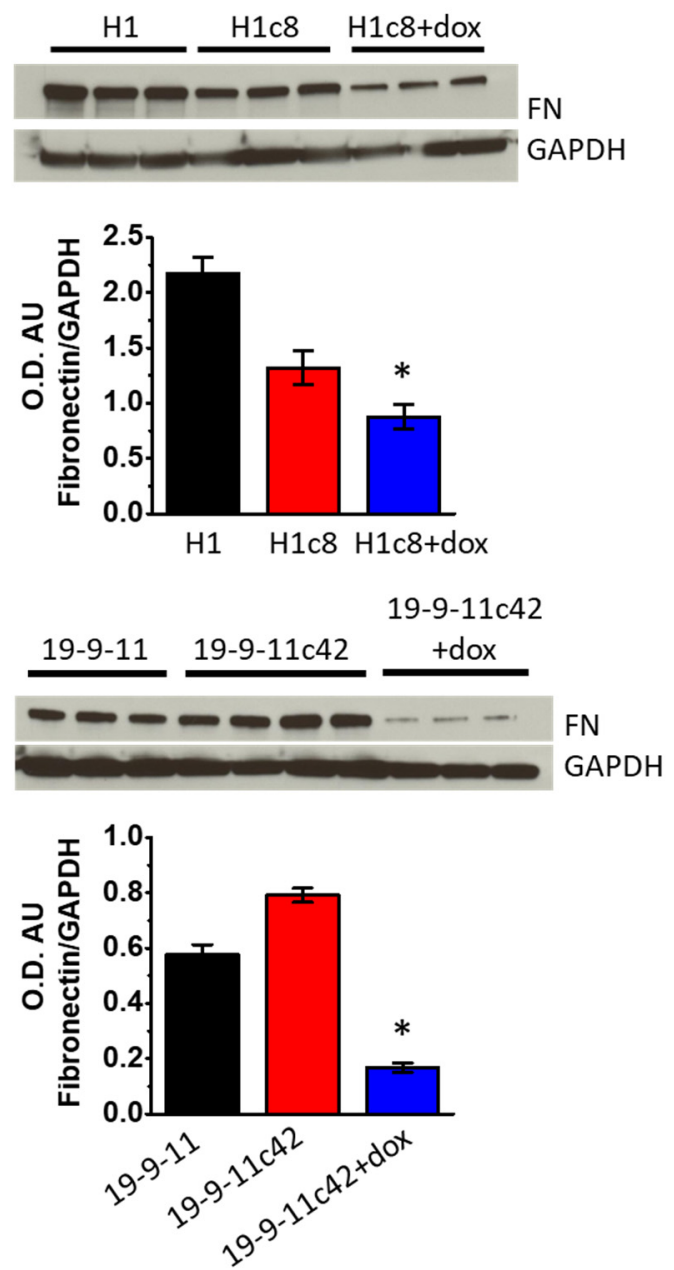

Supplementary Fig. S7 Characterization of hPSC FN1 knockdown clones. A Doxycycline induced mCherry expression in the hPSC culture of H1 FN1 knockdown clone. Scale bar is $200 \mu \mathrm{m}$. B Phase contrast and immuno-fluorescence images of FN expression in doxycycline induced hPSC culture of H1 FNl knockdown clone. Scale bar is $200 \mu \mathrm{m}$. C Quantatitave western blot of FN expression in hPSC culture of $\mathrm{H} 1$ and 19-9-11 FN1 knockdown clones. $\mathrm{N}=3$ biological replicates. $* \mathrm{P}<0.05$, one-way ANOVA with post-hoc Bonferroni and Tukey test. 
A

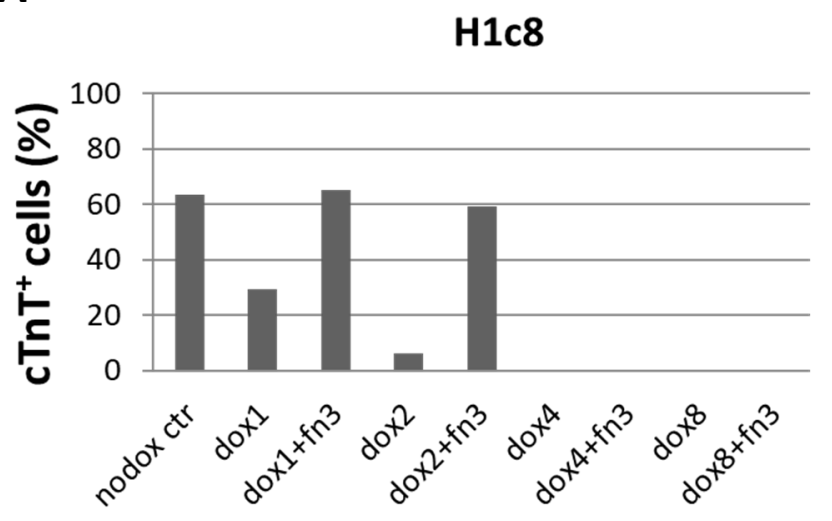

H1c33

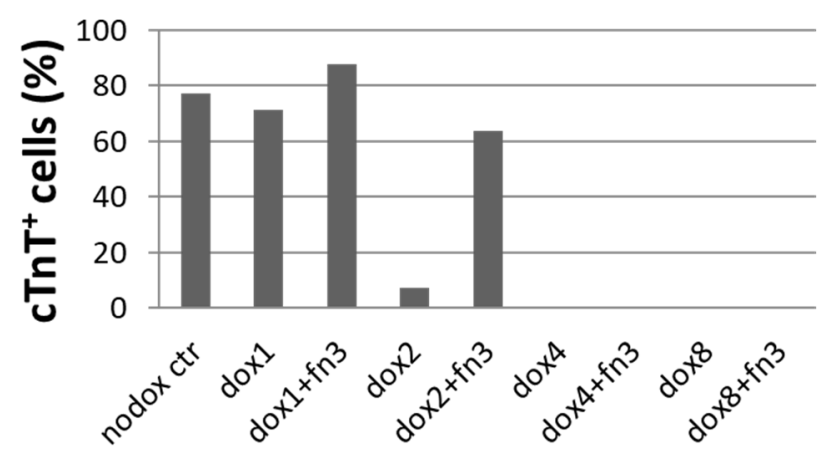

B

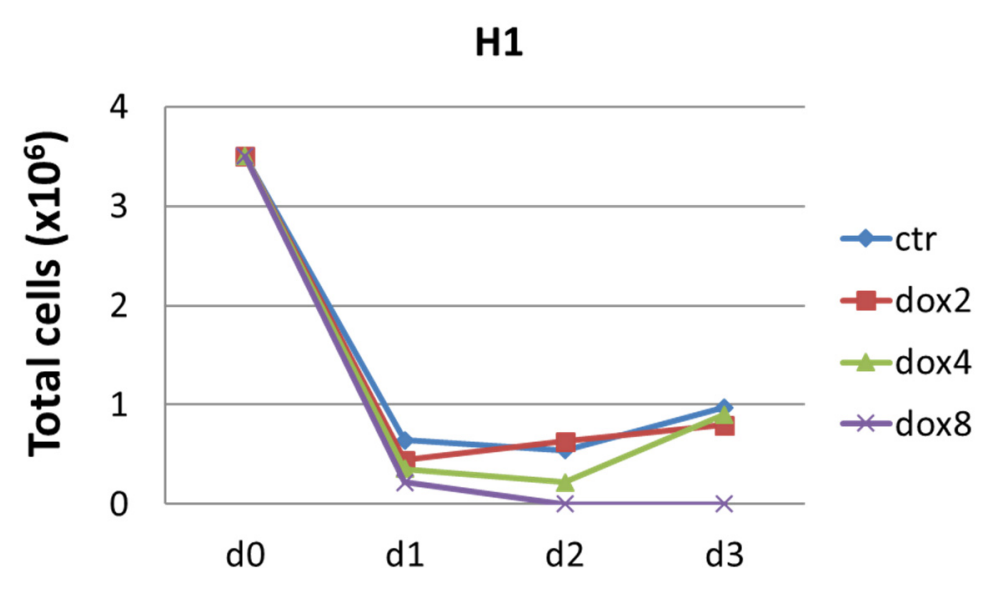

Supplementary Fig. S8 FN

knockdown at day $0-1$ of the cardiac differentiation is doxycycline concentration

- dox do-1 dependent. A cTnT ${ }^{+}$cells measured by flow cytometry at 15 days of differentiation of the FN1 knockdown clones (H1c8 and H1c34) showing the effect of FN knockdown at day $0-1$ at different concentrations of dox and the - dox do-1 effect of adding exogenous $\mathrm{FN}$ at each concentration of dox. Dox concentration higher than $4 \mathrm{ug} / \mathrm{ml}$ caused significant cell death, and cardiac differentiation could not be rescued by exogenous FN.

B The total cells of the regular H1 line in the same cardiac differentiation at day 0-3 when dox was added at day 0 -

1. Dox concentrations of 0 (nodox ctr), 1 (dox1), 2 (dox2), 4 (dox4) and 8 (dox8) $\mathrm{ug} / \mathrm{ml}$ were tested. $+\mathrm{fn}$ indicates exogenous FN (3ug $\left./ \mathrm{cm}^{2}\right)$ was added at day 0 1 for each concentration of dox group. 


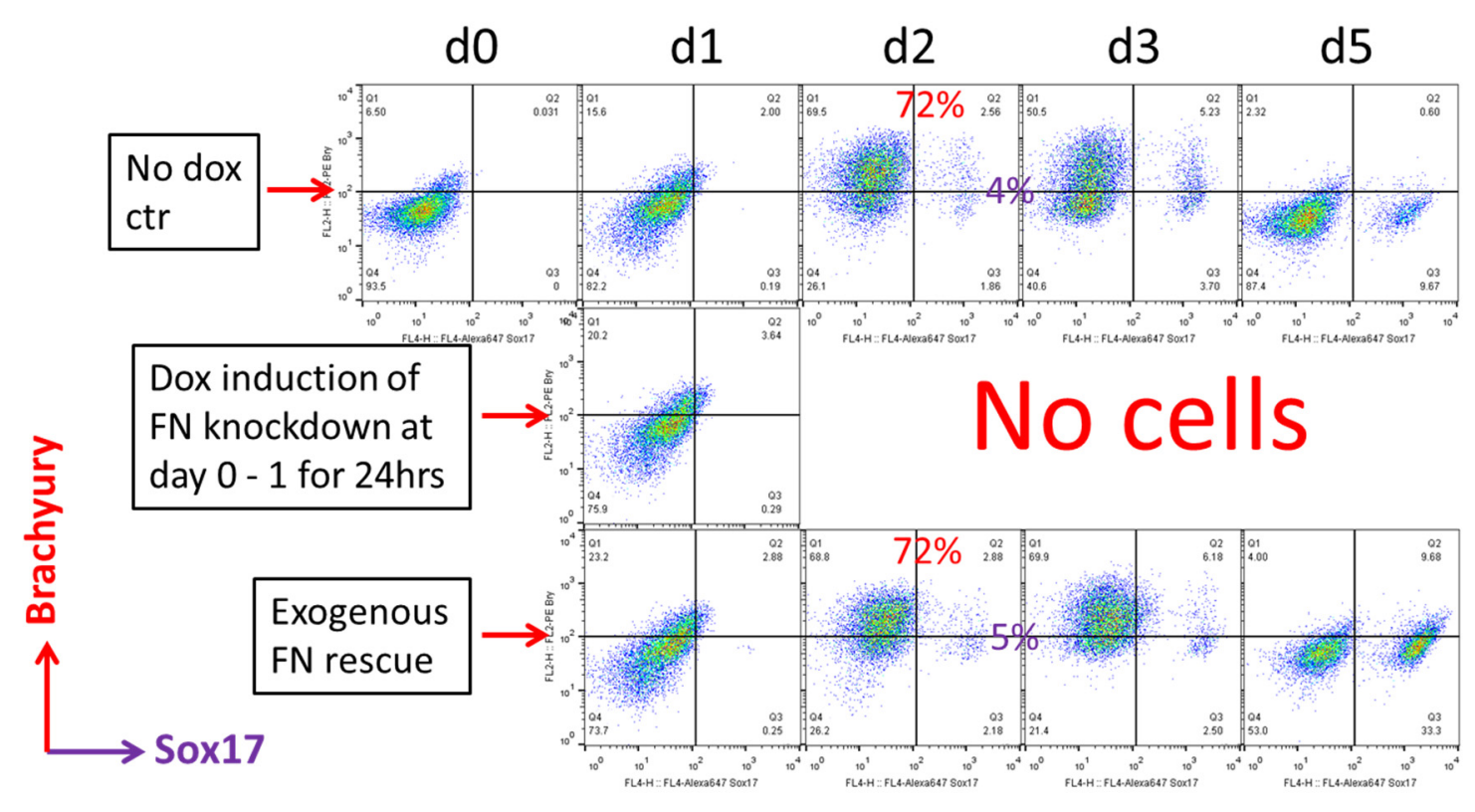

Supplementary Fig. S9 Flow cytometry of co-labeling the cells with Brachyury and Sox17 antibodies of the 19-9-11 FN knockdown clone in the cardiac differentiation time course of day 0-5 at the no dox control, dox induction at day 0-1 and dox induction at day 0-1 with adding exogenous FN conditions.

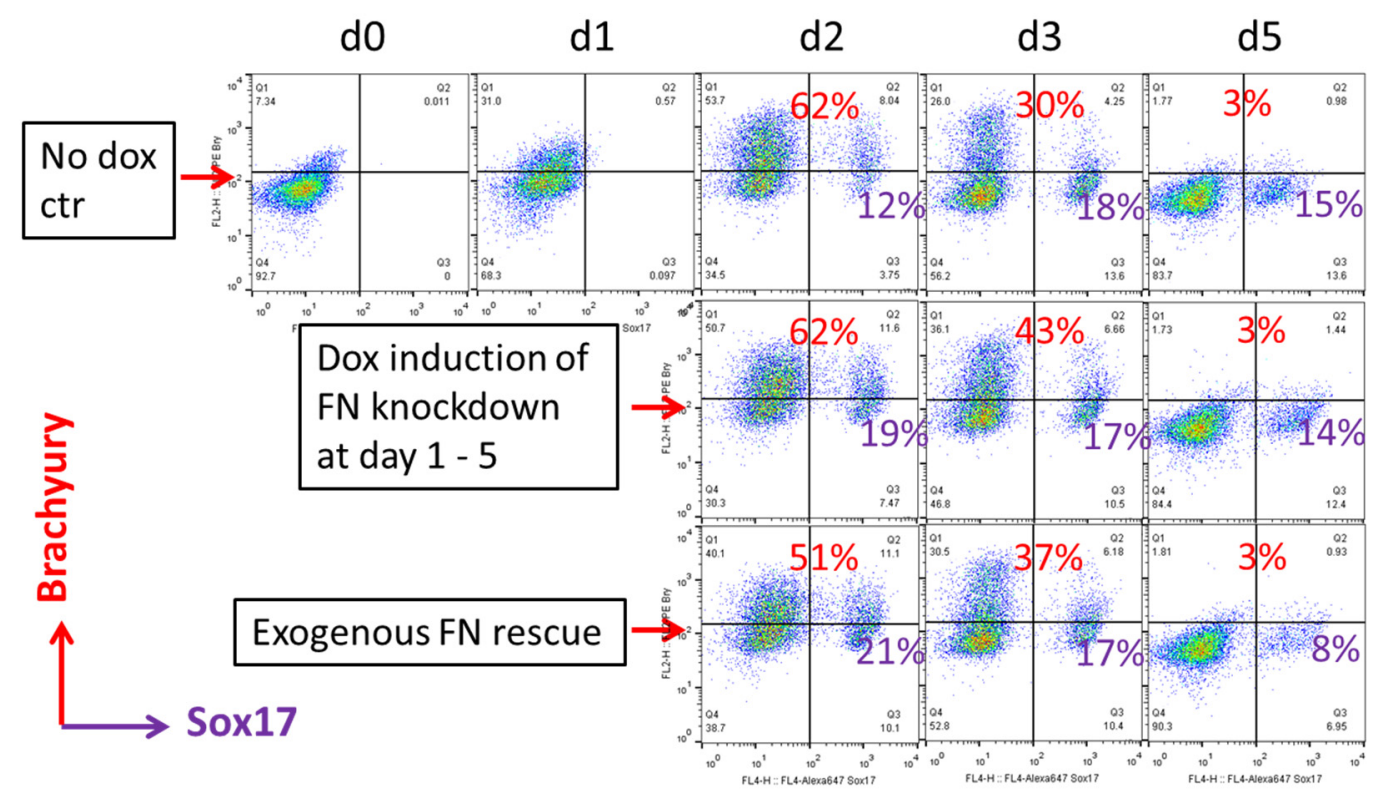

Supplementary Fig. S10 Flow cytometry of co-labeling the cells with Brachyury and Sox17 antibodies of the H1 FN knockdown clone in the cardiac differentiation time course of day 05 at the no dox control, dox induction at day 1-5 and dox induction at day 1-5 with adding exogenous FN conditions. 


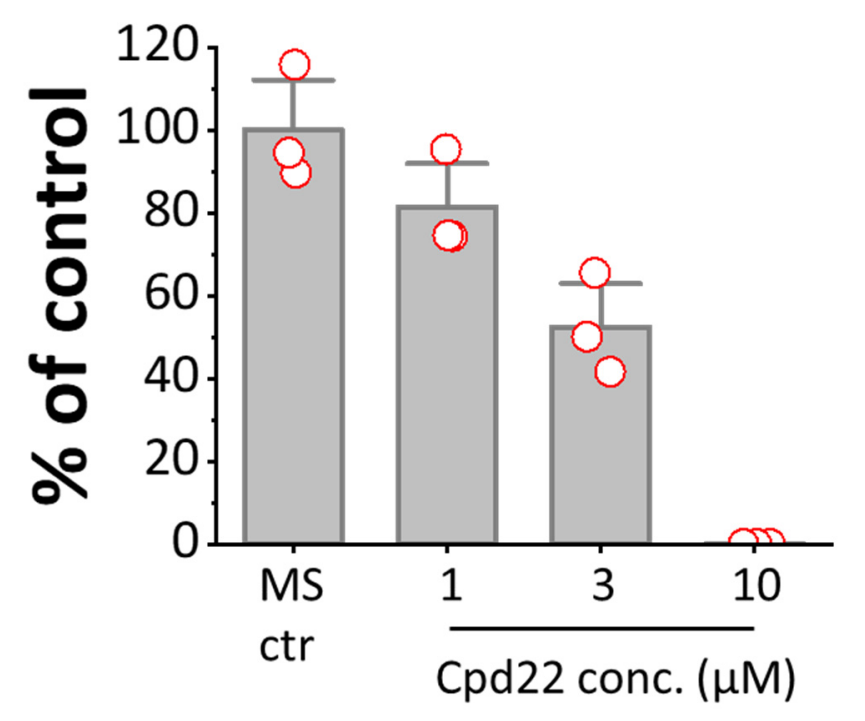

Supplementary Fig. S11 The ILK inhibitor, Cpd22 was added at day 0 at concentrations of $0,1,3$ and $10 \mathrm{uM}$ in the matrix sandwich protocol. Cardiac differentiation was measured by flow cytometry of the $\mathrm{cTnT}^{+}$ cells at 15 days differentiation. The $\mathrm{cTnT}^{+}$ cells in each group was normalized to the control and shown as $\%$ of control on the plot. $\mathrm{N}=3$ biological replicates. Data are form H1 ESC line.

\section{ESC H1}

iPSC DF19-9-11T

Lentivirus (rtTA+Neo; $\stackrel{\text { Split }}{\longrightarrow}$ FN1 shRNA+mCherry) infection, 24-42hrs
Recovery in Neo sel, 11 days mTeSR1, 48hrs

Split, freeze down cells

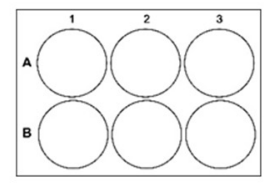

$6 w$, single cell, low density seeding
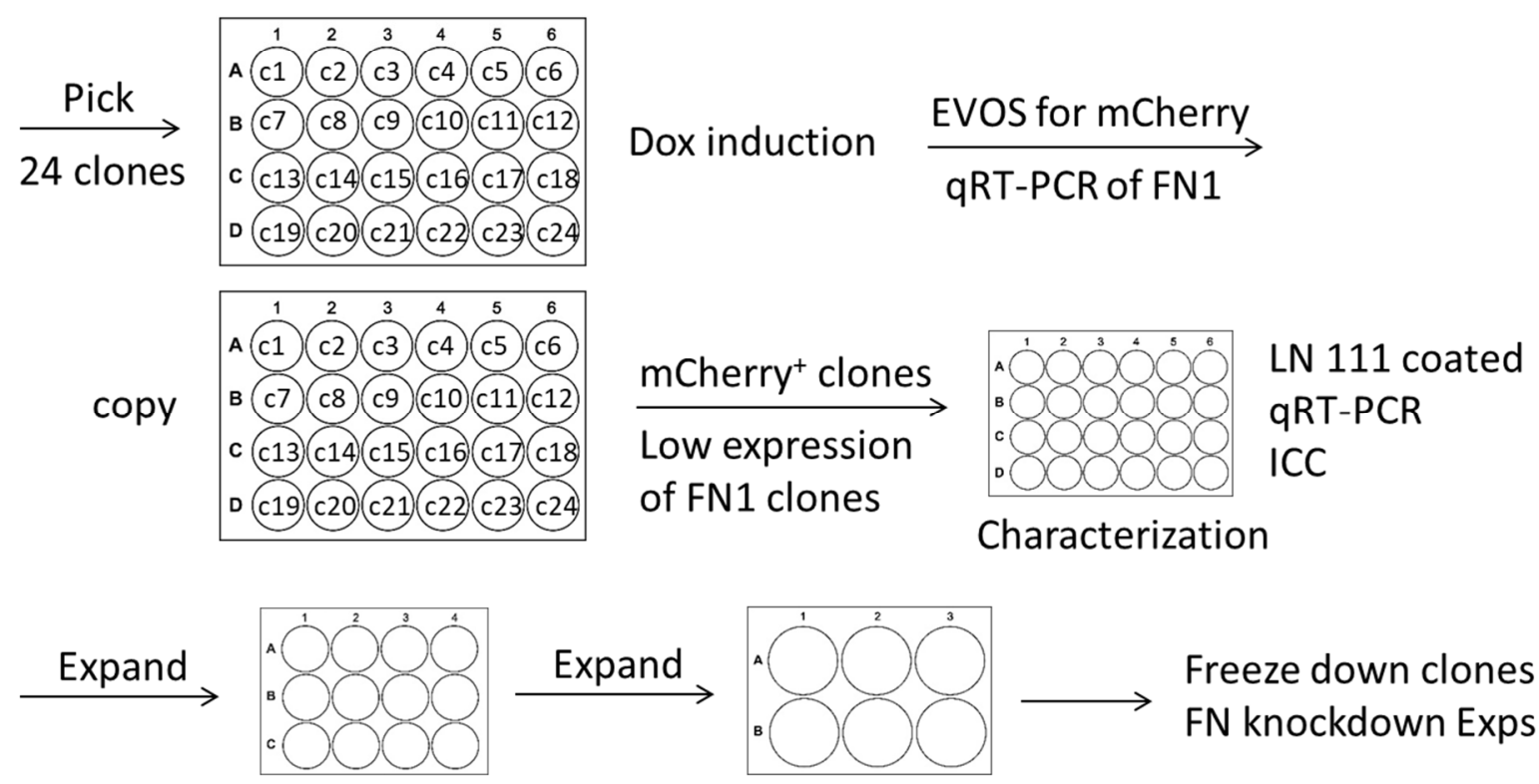

Supplementary Fig. S12 The cloning strategy in generation of inducible FN1 knockdown hPSC clones 
$19-9-11 p 45$

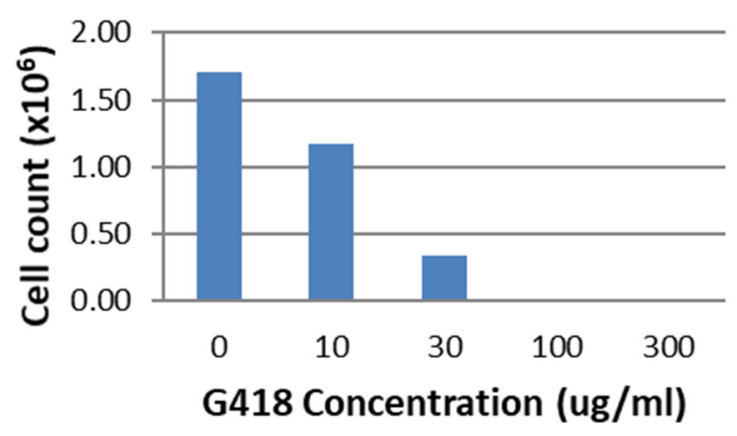

Hp44

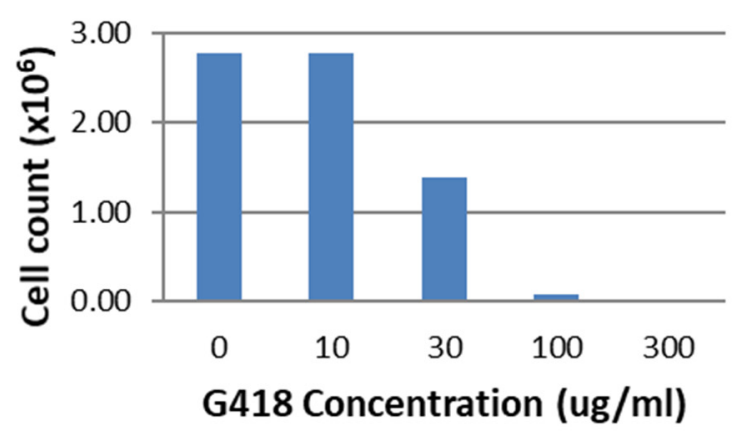

19-9-11p46

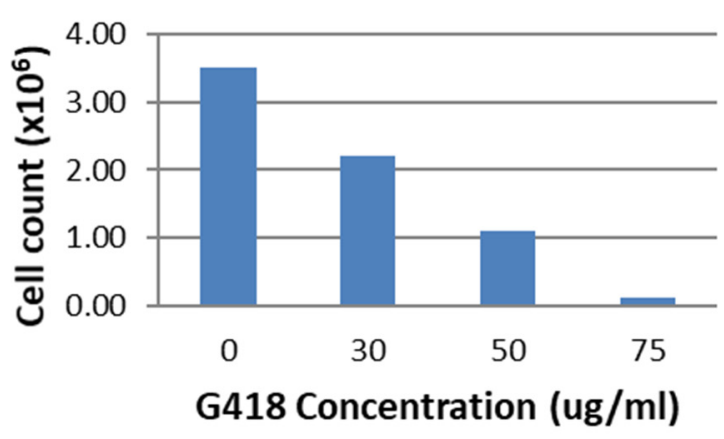

H1p45

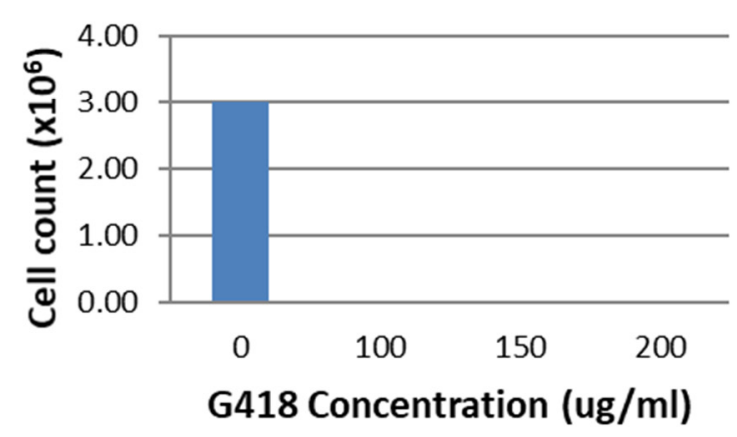

Supplementary Fig. S13 The concentrations of G418 test for neo-resistant selection for hPSC lines. 
Supplementary Table 1. Primary antibodies used in flow cytometry (FC) and immunocytochemistry (ICC)

\begin{tabular}{|c|c|c|c|c|}
\hline Human/Mouse Brachyury PE-conjugated & R\&D (IC2085P) & Goat & IgG & $10 \mathrm{ul} / 1 \times 10^{6}$ cells $(\mathrm{FC})$ \\
\hline $\begin{array}{l}\text { Alexa Fluor } 647 \text { Mouse Anti-Human Sox17 } \\
\text { (Clone P7-969) }\end{array}$ & $\mathrm{BD}(562594)$ & Mouse & $\mathrm{IgG}_{1}$ & $5 \mathrm{ul} / 1 \times 10^{6}$ cells $(\mathrm{FC})$ \\
\hline Human/Mouse Brachyury Antibody & R\&D (AF2085) & Goat & $\operatorname{IgG}$ & $5 \mathrm{ug} / \mathrm{ml}(\mathrm{ICC})$ \\
\hline Troponin T, Cardiac Isoform Ab-1 (Clone 13-11) & $\begin{array}{l}\text { ThermoFisher } \\
\text { Scientific (\#MS- } \\
\text { 295-P) }\end{array}$ & Mouse & $\mathrm{IgG}_{1}$ & $\begin{array}{l}\text { 1:200 dilution }(\mathrm{FC}, \\
\mathrm{ICC})\end{array}$ \\
\hline Purified Mouse Anti-Fibronectin (Clone 10) & $\mathrm{BD}(61007)$ & Mouse & $\mathrm{IgG}_{1}$ & 1:100 dilution (ICC) \\
\hline Anti-Laminin & $\begin{array}{l}\text { Sigma-Aldrich } \\
\text { (L9393) }\end{array}$ & Rabbit & $\mathrm{IgG}$ & 1:500 dilution (ICC) \\
\hline Oct-3/4 (C-10) & $\begin{array}{l}\text { Santa Cruz } \\
\text { (sc-5279) }\end{array}$ & Mouse & $\mathrm{IgG}_{2 \mathrm{~b}}$ & 1:100 dilution (ICC) \\
\hline Anti-SSEA4 antibody [MC813-70] & $\begin{array}{l}\text { Abcam } \\
\text { (ab16287) }\end{array}$ & Mouse & $\mathrm{IgG}_{3}$ & 1:200 dilution (ICC) \\
\hline
\end{tabular}

Supplementary Table 2. Primers for quantitative RT-PCR

Genes

GAPDH

SNAIL1

SNAIL2

TWIST1

VIM

FN1

CDH1

$\mathrm{CDH} 2$

GSC

MIXL1

SOX17

TBXT

MESP1

ISL1

NKX2-5

GATA4

\section{TaqMan ${ }^{\circledR}$ Gene Expression Assay ID}

Hs99999905_m1

Hs00195591_m1

Hs00950344_m1

Hs00361186 m1

Hs00185584_m1

Hs01549976_m1

Hs01023894_m1

Hs00983056 m1

$\mathrm{Hs} 00418279 \mathrm{~m} 1$

Hs00430824_g1

Hs00751752_s1

Hs00610080_m1

$\mathrm{Hs} 00251489-\mathrm{m} 1$

Hs00158126 m1

Hs00231763_m1

Hs00171403_m1 